\title{
An overview of the ant fauna (Hymenoptera: Formicidae) of the state of Maranhão, Brazil
}

\author{
Lívia Pires do Prado ${ }^{1,6}$; Rodrigo Machado Feitosa ${ }^{2,7}$; Stefania Pinzón Trianaa, ; Jhonatan Andrés Muñoz Gutiérrez, \\ Guillaume Xavier Rousseau ${ }^{3,10}$; Raimunda Alves Silva ${ }^{4,11}$; Glécio Machado Siqueira ${ }^{4,12}$; \\ Ciro Líbio Caldas dos Santos ${ }^{5,13}$; Francisco Veras Silva ${ }^{5,14}$; Thiago Sanches Ranzani da Silva, ${ }^{2,15}$; \\ Alexandre Casadei-Ferreira ${ }^{2,16}$; Rogério Rosa da Silva ${ }^{1,17}$ \& Joudellys Andrade-Silva,18
}

\footnotetext{
${ }^{1}$ Museu Paraense Emílio Goeldi (MPEG), Coordenação de Ciências da Terra e Ecologia (COCTE), Curitiba, PR, Brasil.

${ }^{3}$ Universidade Estadual do Maranhão (UEMA), Programa de Pós-Graduação em Agroecologia. São Luís, MA, Brasil.

${ }^{4}$ Universidade Federal do Maranhão (UFMA), Departamento de Geociências (DEGEO). São Luís, MA, Brasil.

${ }^{5}$ Universidade Federal do Maranhão (UFMA). Imperatriz, MA, Brasil.

${ }^{6}$ ORCID: http://orcid.org/0000-0003-1819-8767. E-mail: livia.pires7@gmail.com (corresponding author)

7 ORCID: http://orcid.org/0000-0001-9042-0129. E-mail: rsmfeitosa@gmail.com

${ }^{8}$ ORCID: http://orcid.org/0000-0002-7160-0826. E-mail: stefaniapt@gmail.com

9 ORCID: http://orcid.org/0000-0002-2090-2226. E-mail: energiaselvatica@gmail.com

${ }^{10}$ ORCID: http://orcid.org/0000-0002-2482-4376. E-mail: guilirous@yahoo.ca

${ }^{11}$ ORCID: http://orcid.org/0000-0002-0380-8190. E-mail: ray-234@hotmail.com

${ }^{12}$ ORCID: http://orcid.org/0000-0002-3513-2658. E-mail: gleciosiqueira@hotmail.com

${ }^{13}$ ORCID: http://orcid.org/0000-0002-0987-3620. E-mail: cirolb@gmail.com

${ }^{14}$ ORCID: http://orcid.org/0000-0003-0057-6467. E-mail: fveras_silva@hotmail.com

${ }^{15}$ ORCID: http://orcid.org/0000-0002-4239-1500. E-mail: tsranzanidasilva@gmail.com

${ }^{16}$ ORCID: http://orcid.org/0000-0002-2977-8348. E-mail: alexandrefrreira@gmail.com

${ }^{17}$ ORCID: http://orcid.org/0000-0002-0599-2155.E-mail: rogeriorosas@gmail.com

${ }^{18}$ ORCID: http://orcid.org/0000-0002-5393-6502.E-mail: joudellys@gmail.com
} Laboratório de Morfologia e Ecologia Funcional de Formigas (AntMor), Programa de Pós-Graduação em Zoologia (PPGZO0L). Belém, PA, Brasil.

2 Universidade Federal do Paraná (UFPR), Departamento de Zoologia (DZO0), Laboratório de Sistemática e Biologia de Formigas (LSBF).

\begin{abstract}
The state of Maranhão, located in northeastern Brazil, comprises three biomes: Amazonian, Caatinga, and the Cerrado. To date, 99 ant species have been recorded in the literature from the state. In the present work, we provide for the first time a profile of the ant fauna in the state based on data from the historical literature and Brazilian institutional collections. The updated records on ant diversity for the state of Maranhão revealed a total of 279 species, belonging to 71 genera and 10 subfamilies. In total, 180 species are recorded for the first time in the state, of which four species recorded for the first time in Brazil. In summary, apart from documenting the ant fauna of the region, these results provide a basis for further studies and may contribute to future conservation efforts for the biomes present in this complex landscape.
\end{abstract}

Key-Words. Distribution, Amazon, Caatinga, Cerrado, Checklist.

\section{INTRODUCTION}

Understanding the distribution of species is essential to determine regional and global patterns of biodiversity (Dalzochio et al., 2018). In this sense, taxonomic inventories contribute to characterize areas of endemism, reveal taxonomic novelties and improve scientific collections (Moura et al., 2014; Freitas et al., 2017). Further, the analysis of species distribution databases can help to identify gaps in sampling and species records, and can also be used in macroecological studies, species distribution modeling and to promote conservation strategies (Gasper et al., 2016).

Maranhão is a northeastern state in Brazil and comprises a total area of $329,642.170 \mathrm{~km}^{2}$ (IBGE, 2018). Its political boundaries are the Atlantic Ocean to the north, the state of Tocantins to the south, the state of Piauí to the east, and the state of Pará to the west (Chaves et al., 2016). The state 
is located in a heterogeneous landscape area under the influence of three biomes: Amazon, Cerrado, and the Caatinga. The vegetation cover - encompassing 14 different vegetation types - reflects the transition between super-humid and semi-arid climates (Santos et al., 2010; IBGE, 2018).

Similar to other states in Brazil, Maranhão has suffered with high human impact, mainly from the early 1960s, through the construction of highways, agricultural and mining projects (Celentano et al., 2017). Impacts include large-scale forest conversion to pasture or by "babaçu" palm trees (Orbignya phalerata Mart.) (Santos et al., 2010), and the expansion of agroindustry has converted large natural areas into grain crops (Brasil, 2009; Santos et al., 2010). In addition, other human activities, such as occupation, recreation and tourism (Chaves et al., 2016) have also a negative impact and have caused severe loss of biodiversity, resulting in drastic changes of the landscape.

The biodiversity of Maranhão is extremely diverse (Chaves et al., 2016; Desidério et al., 2017). Compared to other Brazilian states, however, the ant diversity is poorly known. The most recent information on ant species diversity in the state recorded 99 species, belonging to 37 genera and seven subfamilies (Janicki et al., 2016). This represents about $1 / 4$ of ant diversity in the state of Goiás and $35 \%$ of the ant species richness described for the state of Mato Grosso do Sul (Janicki et al., 2016), two other Brazilian states comparable in size to Maranhão.

Since the end of the $20^{\text {th }}$ century, collective efforts of several research groups, carring out inventories in different areas and employing complementary sampling methodologies, resulted in a significant increase in our

Table 1. List of ant collections consulted in this study.

\begin{tabular}{l} 
Repositories \\
\hline Coleção Entomológica, Museu Paraense Emílio Goeldi, Pará, Brazil \\
Laboratório de Biologia, Universidade Federal do Maranhão, \\
Campus Imperatriz, Maranhão, Brazil \\
Laboratório de Entomologia e Vetores, Universidade Federal do Maranhão, \\
Campus São Luís, Maranhão, Brazil \\
Laboratório de Hymenoptera, Museu de Zoologia da Universidade de São Paulo, \\
São Paulo, Brazil \\
Laboratório de Artrópodes e Microbiologia do Solo, Universidade Estadual do Maranhão, \\
Campus São Luís, Maranhão, Brazil \\
Laboratório de Solos e Meio Ambiente, Universidade Federal do Maranhão, \\
Campus São Luís, Maranhão, Brazil
\end{tabular}

knowledge about ant diversity in this state. Thus, the aim of this study is to present an updated list of the ant species in the state of Maranhão, considering recent field expeditions as well as material deposited in the main Brazilian ant collections. We also discuss some relevant aspects about the profile of the ant fauna, recovering the history of ant studies historically carried out in the state. Overall, our findings should be of great help in creating measures for species preservation and species recovery plans and represent the basis for future research.

\section{MATERIAL AND METHODS}

\section{Data from collections and literature}

We listed material obtained from six Brazilian ant collections (Table 1), which have historically acted as main depositary institutions for samples collected in the state of Maranhão. We also compiled data from literature, including collection events focused on partial surveys of Maranhão ant fauna (Table 2).

\section{Identifications and taxonomic validation}

Ants were identified by the authors of the present study using taxonomic keys, comparing specimens with myrmecological collections, or by sending them to specialists (see "Acknowledgements"). The final list containing all specimens was verified by authors of this study (JAS, LPP and RMF). Species with dubious identification were carefully examined and, when necessary, have been removed from final data set.

\section{Distribution and maps}

The biomes present in Maranhão are the Amazon Forest, characterized by tall trees and periodic to permanently flooded plains; this biome is present in the north and, essentially, in the west portion of the state. The Cerrado covers the south, central and northeast areas of the state, formed by open grasslands (Cerrado aberto) to patches of dense vegetation (Cerradão). Finally, Maranhão presents a small and fragmented portion of

Table 2. References and their respective areas that have records of ants collected in the state of Maranhão.

\begin{tabular}{ll}
\hline \multicolumn{1}{c}{ Context } & \multicolumn{1}{c}{ Source } \\
\hline Behavioral & Wheeler, 1922; Monnin et al., 2003; Silva, 2007; Pereira et al., 2014 \\
Ecological & Brandão et al., 2011; Ramos et al., 2015; Silva et al., 2017; Gutiérrez et al., 2017 \\
Forensic & Andrade-Silva et al., 2015; Pereira et al., 2017 \\
Genetics & Wauters et al., 2018 \\
Inventory & Dáttilo et al., 2012 \\
Occurrence & Dáttilo et al., 2010 \\
Sanitary & Carvalho et al., 2011; Silva et al., 2012; Lima et al., 2013 \\
Taxonomic & Forel, 1904; Mann, 1916; Gonçalves, 1942, 1947; Borgmeier, 1955; Kempf, 1959, 1960a, 1960b; Gonçalves, 1961; Kempf, 1964, 1968, 1971, 1972a, 1972b, 1975; Watkins, 1976; \\
& Ward, 1989; Brandão, 1991; De Andrade \& Baroni Urbani, 1999; Ward, 1999; Cuezzo, 2000; Longino \& Snelling, 2002; Ward \& Downie, 2005; Shoemaker et al., 2006; Wild \& Cuezzo, 2006; \\
& Feitosa et al., 2007; Ward, 2007; Wild, 2007; Feitosa et al., 2008; Lattke, 2011; Fernandes et al., 2014; Brandão et al., 2015; Johnson, 2015; Ulysséa et al., 2015, 2017; \\
\hline & Jesovnik \& Schultz, 2017; Dias \& Lattke, 2019 \\
\hline
\end{tabular}


the Caatinga biome, in the extreme east of the state, characterized by the presence of bushy vegetation with deep roots, cacti and bromeliads (Spinelli-Araujo et al., 2016).

We used shapefiles from the state of Maranhão made available by the Ministério do Meio Ambiente (MMA) (http://mapas.mma.gov.br/i3geo/datadownload.htm\#). We used a classification in "meso-regions" pre-established by the government agency, in order to describe and discuss our results. We also used shapefiles provided by MMA for the three main biomes present in the state, to overlap sampling points and the main ecosystems in Maranhão.

For the confirmation of sampled sites (Table 3) and maps preparation, the geographical coordinates, when not available on the specimens' label, were obtained from the IBGE (2011) or georeferenced using Google Earth Pro. In those cases, because we did not have access to the exact point of the sample site, we adapted a classification by the IBGE. Whenever the IBGE classified a municipality covering two biomes, we used the "transition" term after the government classification. For instance, the municipality of Imperatriz, which is classified by IBGE as "Amazon/Cerrado" biomes, becomes for the purpose of this study, "Amazon-Cerrado transition". For specific sites and localities for which names have been historically altered, we consulted Vanzolini \& Papavero (1968) and Vanzolini (1992). The geographical records were mapped using QGIS v2.18.2 (QGIS Development Team, 2019).

\section{RESULTS}

Based on data from Brazilian collections (Table 1) and published literature (Table 2), we recorded a total of 279 ant species for the state of Maranhão, belonging to 71 genera and 10 subfamilies (Table 4), and sampled across 65 localities (Table 3 ). The subfamily Myrmicinae was the most diverse, with 126 species, followed by Ponerinae (36 species), Formicinae (35 species), Dolichoderinae (27 species), Ectatomminae (25 species), Pseudomyrmecinae (16 species), Dorylinae (10 species), Amblyoponinae (2 species), and Paraponerinae and Proceratiinae (1 species each).

The majority of records (214 species) was concentrated along the Amazon region, followed by the Cerrado (129 species), the Amazon-Cerrado transition regions (80 species) and finally the Cerrado-Caatinga transition region where only one species was recorded (Fig. 1). A total of 180 ant species were recorded for the first time in the state, and four species were recorded for the first time in Brazil (Table 4).

\section{DISCUSSION}

The first expeditions focused on studying the ant fauna of the state of Maranhão were performed in the late 1940s, with collections in the Cerrado areas undertaken by the myrmecologists Cincinnato Gonçalves and Walter
W. Kempf. During the next three decades, collections by researchers, enthusiasts, and professional collectors had pursued the same goal - discovering new taxa and increasing the coverage of ants in scientific collections (Kempf, 1972a). Differently, from the 1980s until the beginning of the $21^{\text {st }}$ century, the main purpose of the expeditions was to carry out environmental impact assessment programs (Brandão et al., 2011). On the other hand, from the late $20^{\text {th }}$ century, with the hiring of researchers at universities in the state of Maranhão, several expeditions have been conducted focusing on ecological studies and reporting faunal inventories (Ramos et al., 2015; Gutiérrez et al., 2017; Silva et al., 2017).

Museums, scientific collections, and historical published literature all contain important information on species distributions recorded as presence data (Newbold, 2010). The accuracy of the distribution data is important for several applications in biology and for species conservation planning (Graham et al., 2008). Despite the concern to accurately document of species distribution that began in the first half of the $19^{\text {th }}$ century (Vanzolini, 2004), for the ants this occurred in the second half of the $20^{\text {th }}$ century. In the case of the records analysed in this work, the specific localities and geographic coordinates became available in the late $20^{\text {th }}$ century.

Most of the ant records for the state of Maranhão remained unavailable to the specialized public for a long time, while many other records remained unidentified at a specific level. In this sense, our study has analyzed both the material deposited in Brazilian collections (Table 1) and the records in the published literature (Table 2), revealing that $64 \%$ of species were recorded in the state for the first time. Further, we made an additional effort

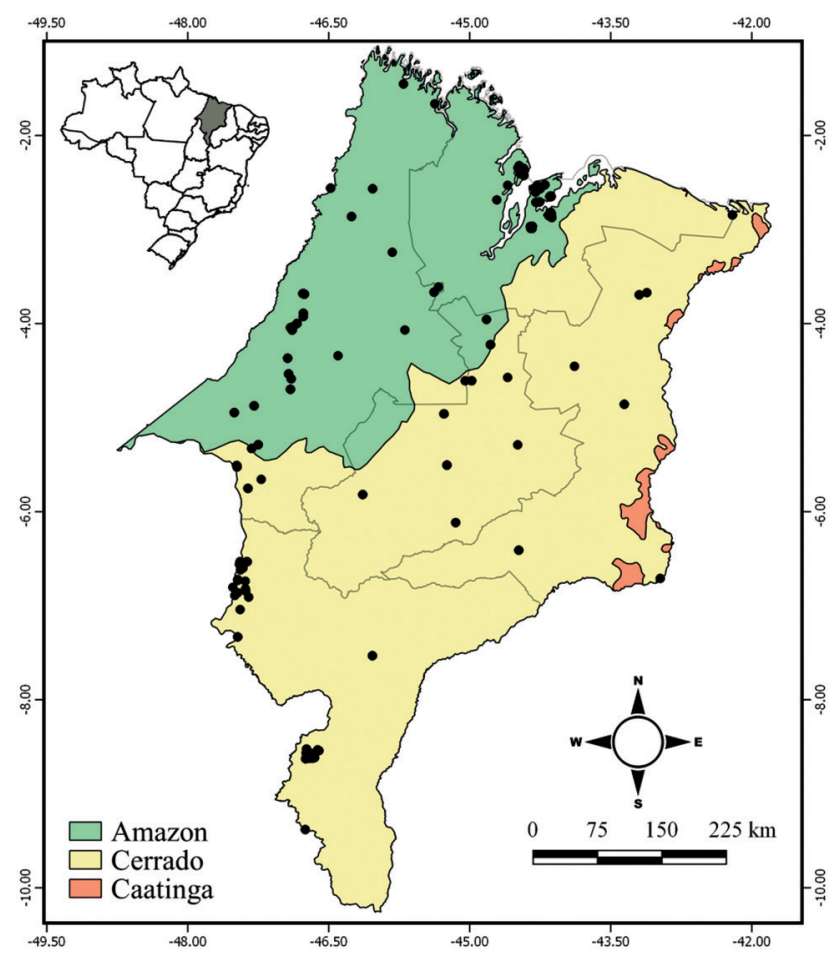

Figure 1. Map of the state of Maranhão showing its location in Brazil. Black points indicate the sampling sites within the state that were georeferenced and recorded in the literature and collections according to biomes. 
Table 3. Information from the sampled sites for the state of Maranhão. The abbreviations are as follows: (Am) Amazon, (Ce) Cerrado, (ACT) Amazon-Cerrado transition, (CCT) Cerrado-Caatinga transition. $\left(^{*}\right)$ For the geographic coordinates attributed in this work.

\begin{tabular}{|c|c|c|c|}
\hline Locality & Coordinate & Physiognomy & Code \\
\hline 15 km E of Canindé, Aldeia Araçu, Igarapé Gurupi-Uma & $02^{\circ} 34^{\prime} S, 46^{\circ} 02^{\prime} \mathrm{W}^{*}$ & $\mathrm{Am}$ & 1 \\
\hline Açailândia & $04^{\circ} 52^{\prime} 30^{\prime \prime} \mathrm{S}, 47^{\circ} 17^{\prime} 40^{\prime \prime} \mathrm{W}$ & Am & 2 \\
\hline Açailândia, Fazenda Pedro Maranhão & $04^{\circ} 56^{\prime} 48^{\prime \prime} \mathrm{S}, 47^{\circ} 30^{\prime} 17^{\prime \prime} \mathrm{W}$ & Am & 3 \\
\hline Açailândia, Horto Fazenda Pompéia & $04^{\circ} 52^{\prime} 30^{\prime \prime} \mathrm{S}, 47^{\circ} 17^{\prime} 40^{\prime \prime} \mathrm{W}$ & Am & 4 \\
\hline Alcântara & $02^{\circ} 20^{\prime} 56^{\prime \prime} S, 44^{\circ} 29^{\prime} 01^{\prime \prime} \mathrm{W}$ & Am & 5 \\
\hline Alcântara, Só Assim & $02^{\circ} 20^{\prime} 53.48^{\prime \prime} \mathrm{S}, 44^{\circ} 28^{\prime} 50.71^{\prime \prime} \mathrm{W}$ & Am & 6 \\
\hline Aldeia do Ponto & $06^{\circ} 07^{\prime} 01.21^{\prime \prime} \mathrm{S}, 45^{\circ} 08^{\prime} 59.99^{\prime \prime} \mathrm{W}^{*}$ & Ce & 7 \\
\hline Alto Turiaçu & $01^{\circ} 39^{\prime} 46^{\prime \prime} \mathrm{S}, 45^{\circ} 22^{\prime} 19^{\prime \prime} \mathrm{W}^{*}$ & Am & 8 \\
\hline Alto Turiaçu, Aldeia Gurupiuna & $02^{\circ} 51^{\prime} 44.66^{\prime \prime} \mathrm{S}, 46^{\circ} 15^{\prime} 29.79^{\prime \prime} \mathrm{W}^{*}$ & Am & 9 \\
\hline Bacabal & $04^{\circ} 13^{\prime} 30^{\prime \prime} \mathrm{S}, 44^{\circ} 46^{\prime} 48^{\prime \prime} \mathrm{W}^{*}$ & ACT & 10 \\
\hline Balsas & $08^{\circ} 34^{\prime} 19.6^{\prime \prime} S, 46^{\circ} 42^{\prime} 28.2^{\prime \prime} \mathrm{W}$ & Ce & 11 \\
\hline Balsas, Córrego Xupé & $07^{\circ} 31^{\prime} 58^{\prime \prime S}, 46^{\circ} 02^{\prime} 09^{\prime \prime} \mathrm{W}$ & Ce & 12 \\
\hline Balsas, Gerais de Balsas & $08^{\circ} 34^{\prime} 06^{\prime \prime} \mathrm{S}, 46^{\circ} 42^{\prime} 38^{\prime \prime} \mathrm{W}$ & $\mathrm{Ce}$ & 13 \\
\hline Balsas, Gerais de Balsas, Rio Mandacaru & $08^{\circ} 32^{\prime} 32^{\prime \prime} S, 46^{\circ} 36^{\prime} 18^{\prime \prime} \mathrm{W}$ & $\mathrm{Ce}$ & 14 \\
\hline Balsas, Mata do Capão do Catulé & $09^{\circ} 22^{\prime} 53.8^{\prime \prime} \mathrm{S}, 46^{\circ} 44^{\prime} 59.3^{\prime \prime} \mathrm{W}$ & $\mathrm{Ce}$ & 15 \\
\hline Barão de Grajaú, Bem Quer & $06^{\circ} 09^{\prime} 24.5^{\prime \prime} \mathrm{S}, 42^{\circ} 58^{\prime} 02.0^{\prime \prime} \mathrm{W}$ & CCT & 16 \\
\hline Barra do Corda & $05^{\circ} 30^{\prime} 21^{\prime \prime} \mathrm{S}, 45^{\circ} 14^{\prime} 34^{\prime \prime} \mathrm{W}^{*}$ & Ce & 17 \\
\hline Bom Jardim & $03^{\circ} 55^{\prime} 32.90^{\prime \prime} S, 46^{\circ} 46^{\prime} 16.33^{\prime \prime} \mathrm{W}$ & $\mathrm{Am}$ & 18 \\
\hline Buriticupu & $04^{\circ} 20^{\prime} 45^{\prime \prime} \mathrm{S}, 46^{\circ} 24^{\prime} 03^{\prime \prime} \mathrm{W}^{*}$ & $\mathrm{Am}$ & 19 \\
\hline Buriticupu, Fazenda Cacique & $04^{\circ} 20^{\prime} 34^{\prime \prime} \mathrm{S}, 46^{\circ} 24^{\prime} 06^{\prime \prime} \mathrm{W}^{*}$ & $\mathrm{Am}$ & 20 \\
\hline Cajazeiras & $02^{\circ} 50^{\prime} 43^{\prime \prime} \mathrm{S}, 42^{\circ} 12^{\prime} 20^{\prime \prime} \mathrm{W}^{*}$ & Ce & 21 \\
\hline Carolina & $07^{\circ} 19^{\prime} 58^{\prime \prime} \mathrm{S}, 47^{\circ} 28^{\prime} 08^{\prime \prime} \mathrm{W}^{*}$ & $\mathrm{Ce}$ & 22 \\
\hline Carolina, Pedra Caída & $07^{\circ} 02^{\prime} 30.39^{\prime \prime} \mathrm{S}, 47^{\circ} 26^{\prime} 35.95^{\prime \prime} \mathrm{W}^{*}$ & Ce & 23 \\
\hline Caxias & $04^{\circ} 51^{\prime} 32^{\prime \prime} S, 43^{\circ} 21^{\prime} 21^{\prime \prime} \mathrm{W}$ & Ce & 24 \\
\hline Centro Novo do Maranhão & $03^{\circ} 40^{\prime} 55.70^{\prime \prime} \mathrm{S}, 46^{\circ} 46^{\prime} 40.73^{\prime \prime} \mathrm{W}$ & Am & 25 \\
\hline Chapadinha, Anapurus & $03^{\circ} 40^{\prime} 19^{\prime \prime} S, 43^{\circ} 06^{\prime} 57^{\prime \prime} \mathrm{W}$ & Ce & 26 \\
\hline Chapadinha, Fazenda Unha de Gato & $03^{\circ} 41^{\prime} 42.3^{\prime \prime} S, 43^{\circ} 11^{\prime} 48.1^{\prime \prime} \mathrm{W}$ & $\mathrm{Ce}$ & 27 \\
\hline Codó & $04^{\circ} 27^{\prime} 18^{\prime \prime} S, 43^{\circ} 53^{\prime} 09^{\prime \prime} \mathrm{W}^{*}$ & $\mathrm{Ce}$ & 28 \\
\hline Estreito & $06^{\circ} 50^{\prime} 45^{\prime \prime} S, 47^{\circ} 23^{\prime} 00^{\prime \prime} \mathrm{W}$ & $\mathrm{Ce}$ & 29 \\
\hline Estreito, Fazenda Itaueiras & $06^{\circ} 31^{\prime} 54.4^{\prime \prime} S, 47^{\circ} 22^{\prime} 16.0^{\prime \prime} \mathrm{W}$ & Ce & 30 \\
\hline Estreito, Fazenda Planalto & $06^{\circ} 35^{\prime} 59.3^{\prime \prime} S, 47^{\circ} 24^{\prime} 50.4^{\prime \prime} \mathrm{W}$ & Ce & 31 \\
\hline Estreito, Ilha do Cabral, Rio Tocantins & $06^{\circ} 31^{\prime} 54.1^{\prime \prime S}, 47^{\circ} 26^{\prime} 32.6^{\prime \prime} \mathrm{W}$ & Ce & 32 \\
\hline Grajaú, Rio Santana & $05^{\circ} 49^{\prime} 08^{\prime \prime} \mathrm{S}, 46^{\circ} 08^{\prime} 20^{\prime \prime} \mathrm{W}^{*}$ & ACT & 33 \\
\hline Gurupi & $04^{\circ} 22^{\prime} 09.04^{\prime \prime} \mathrm{S}, 46^{\circ} 56^{\prime} 16.05^{\prime \prime} \mathrm{W}$ & Am & 34 \\
\hline Imperatriz & $05^{\circ} 30^{\prime} 38^{\prime \prime} \mathrm{S}, 47^{\circ} 28^{\prime} 46^{\prime \prime} \mathrm{W}^{*}$ & ACT & 35 \\
\hline Imperatriz, Bananal & $05^{\circ} 39^{\prime} 26^{\prime \prime} \mathrm{S}, 47^{\circ} 13^{\prime} 07^{\prime \prime} \mathrm{W}^{*}$ & Ce & 36 \\
\hline Imperatriz, Reserva do $50^{\circ}$ Batalhão de Infantaria e Selva & $05^{\circ} 30^{\prime} 37.60^{\prime \prime} \mathrm{S}, 47^{\circ} 28^{\prime} 46.11^{\prime \prime} \mathrm{W}$ & ACT & 37 \\
\hline Imperatriz, Ribeirãozinho & $05^{\circ} 45^{\prime} 11.15^{\prime \prime} \mathrm{S}, 47^{\circ} 21^{\prime} 36.36^{\prime \prime} \mathrm{W}^{*}$ & Ce & 38 \\
\hline Imperatriz, Tocantinópolis & $05^{\circ} 31^{\prime} 33^{\prime \prime} \mathrm{S}, 47^{\circ} 28^{\prime} 33^{\prime \prime} \mathrm{W}^{*}$ & ACT & 39 \\
\hline Itinga do Maranhão & $04^{\circ} 02^{\prime} 05.50^{\prime \prime} \mathrm{S}, 46^{\circ} 52^{\prime} 51.00^{\prime \prime} \mathrm{W}^{*}$ & Am & 40 \\
\hline João Lisboa & $05^{\circ} 19^{\prime} 46.30^{\prime \prime} \mathrm{S}, 47^{\circ} 19^{\prime} 13.00^{\prime \prime} \mathrm{W}$ & $\mathrm{Am}$ & 41 \\
\hline Lago do Junco & $04^{\circ} 36^{\prime} 32^{\prime \prime} \mathrm{S}, 45^{\circ} 02^{\prime} 56^{\prime \prime} \mathrm{W}^{*}$ & ACT & 42 \\
\hline Lago dos Rodrigues & $04^{\circ} 36^{\prime} 32^{\prime \prime} \mathrm{S}, 44^{\circ} 58^{\prime} 48^{\prime \prime} \mathrm{W}^{*}$ & Ce & 43 \\
\hline Lago Verde & $03^{\circ} 57^{\prime} 25^{\prime \prime} \mathrm{S}, 44^{\circ} 49^{\prime} 19^{\prime \prime} \mathrm{W}^{*}$ & $\mathrm{Am}$ & 44 \\
\hline Lagoa Grande do Maranhão & $04^{\circ} 57^{\prime} 38.77^{\prime \prime} \mathrm{S}, 45^{\circ} 16^{\prime} 27.88^{\prime \prime} \mathrm{W}^{*}$ & Ce & 45 \\
\hline Livramento & $02^{\circ} 25^{\prime} 33.19^{\prime \prime} \mathrm{S}, 44^{\circ} 25^{\prime} 26.59^{\prime \prime} \mathrm{W}$ & $\mathrm{Am}$ & 46 \\
\hline Mirador, Parque Estadual do Mirador & $06^{\circ} 24^{\prime} 38.62^{\prime \prime} \mathrm{S}, 44^{\circ} 28^{\prime} 42.24^{\prime \prime} \mathrm{W}$ & $\mathrm{Ce}$ & 47 \\
\hline Pedreiras & $04^{\circ} 34^{\prime} 26^{\prime \prime} S, 44^{\circ} 35^{\prime} 49^{\prime \prime} \mathrm{W}^{*}$ & Ce & 48 \\
\hline Pindaré-Mirim, Sítio Moisés & $03^{\circ} 36^{\prime} 44.00^{\prime \prime} S, 45^{\circ} 19^{\prime} 59.90^{\prime \prime} \mathrm{W}$ & $\mathrm{Am}$ & 49 \\
\hline Presidente Dutra & $05^{\circ} 17^{\prime} 24^{\prime \prime} S, 44^{\circ} 29^{\prime} 24^{\prime \prime} \mathrm{W}$ & Ce & 50 \\
\hline Rio Maracaçumé & $01^{\circ} 27^{\prime} 09^{\prime \prime} \mathrm{S}, 45^{\circ} 42^{\prime} 19^{\prime \prime} \mathrm{W}$ & Am & 51 \\
\hline Rosário & $02^{\circ} 51^{\prime} 11.8^{\prime \prime} \mathrm{S}, 44^{\circ} 09^{\prime} 02.6^{\prime \prime} \mathrm{W}$ & Am & 52 \\
\hline Santa Inês & $03^{\circ} 40^{\prime} 01^{\prime \prime} \mathrm{S}, 45^{\circ} 22^{\prime} 48^{\prime \prime} \mathrm{W}$ & Am & 53 \\
\hline Santa Luzia & $04^{\circ} 04^{\prime} 08^{\prime \prime} S, 45^{\circ} 41^{\prime} 24^{\prime \prime} \mathrm{W}$ & ACT & 54 \\
\hline São Francisco do Brejão & $05^{\circ} 17^{\prime} 19.0^{\prime \prime} \mathrm{S}, 47^{\circ} 15^{\prime} 01.7^{\prime \prime} \mathrm{W}$ & $\mathrm{Am}$ & 55 \\
\hline São José de Ribamar, Sítio Aguahy & $02^{\circ} 38^{\prime} 59.30^{\prime \prime} S, 44^{\circ} 08^{\prime} 49.63^{\prime \prime} \mathrm{W}$ & $\mathrm{Am}$ & 56 \\
\hline São Luís & $02^{\circ} 31^{\prime} 48^{\prime \prime} S, 44^{\circ} 18^{\prime} 10^{\prime \prime} \mathrm{W}^{*}$ & $\mathrm{Am}$ & 57 \\
\hline São Luís, Área de Preservação Ambiental do Itapiracó & $02^{\circ} 31^{\prime} 28.81^{\prime \prime} \mathrm{S}, 44^{\circ} 12^{\prime} 00.33^{\prime \prime} \mathrm{W}$ & Am & 58 \\
\hline São Luís, Campus da Universidade Federal do Maranhão & $02^{\circ} 33^{\prime} 36^{\prime \prime S}, 44^{\circ} 18^{\prime} 33^{\prime \prime} \mathrm{W}$ & Am & 59 \\
\hline São Luís, Maracana, Sítio Mangalho & $02^{\circ} 36^{\prime} 13.39^{\prime \prime} S, 44^{\circ} 17^{\prime} 56.78^{\prime \prime} \mathrm{W}$ & Am & 60 \\
\hline São Luís, Mata do Quebra Pote & $02^{\circ} 42^{\prime} 26.75^{\prime \prime} S, 44^{\circ} 15^{\prime} 23.87^{\prime \prime} \mathrm{W}$ & Am & 61 \\
\hline São Luís, Reserva Alumar & $02^{\circ} 42^{\prime} 39.31^{\prime \prime} S, 44^{\circ} 17^{\prime} 47.40^{\prime \prime} \mathrm{W}$ & Am & 62 \\
\hline São Luís, Reserva da CAEMA & $02^{\circ} 34^{\prime} 01.01^{\prime \prime} S, 44^{\circ} 15^{\prime} 04.03^{\prime \prime} \mathrm{W}$ & $\mathrm{Am}$ & 63 \\
\hline São Mateus & $04^{\circ} 02^{\prime} 24^{\prime \prime} S, 44^{\circ} 28^{\prime} 12^{\prime \prime} \mathrm{W}$ & ACT & 64 \\
\hline Zé Doca & $03^{\circ} 14^{\prime} 34^{\prime \prime} \mathrm{S}, 45^{\circ} 49^{\prime} 26^{\prime \prime} \mathrm{W}^{*}$ & $\mathrm{Am}$ & 65 \\
\hline
\end{tabular}


Table 4. List of taxa recorded in the state of Maranhão and the occurrence data of the species in the literature and localities and biome present in the state. The codes of localities follow Table 3. $\left(^{*}\right)$ new record for Maranhão, $\left({ }^{* *}\right)$ new record for Brazil, (Am) Amazon, (Ce) Cerrado, (ACT) Amazon-Cerrado transition, (CCT) CerradoCaatinga transition.

\begin{tabular}{|c|c|c|c|}
\hline Taxon name & Locality & Biome & Source \\
\hline Amblyoponinae Forel, 1893* & - & - & - \\
\hline Fulakora Mann, 1919* & - & - & - \\
\hline Fulakora degenerata (Borgmeier, 1957)* & 4,41 & $A m$ & Collection \\
\hline Prionopelta Mayr, 1866* & - & - & - \\
\hline Prionopelta antillana Forel, 1909* & 46 & $\mathrm{Am}$ & Collection \\
\hline Dolichoderinae Forel, 1878 & - & - & - \\
\hline Azteca Forel, 1878* & - & - & - \\
\hline Azteca alfari Emery, 1893* & 13,37 & $\mathrm{Ce}, \mathrm{ACT}$ & Collection \\
\hline Azteca chartifex Emery, 1896* & 57 & $\mathrm{Am}$ & Collection \\
\hline Azteca schimperi Emery, 1893* & 57 & Am & Collection \\
\hline Dolichoderus Lund, 1831 & - & - & - \\
\hline Dolichoderus abruptus (Smith, 1858)* & 8 & $\mathrm{Am}$ & Collection \\
\hline Dolichoderus attelaboides (Fabricius, 1775)* & $1,2,56$ & $A m$ & Collection \\
\hline Dolichoderus bidens (Linnaeus, 1758)* & 19 & Am & Collection \\
\hline Dolichoderus bispinosus (Olivier, 1792)* & $5,24,35,37,39$ & $\mathrm{Am}, \mathrm{Ce}, \mathrm{ACT}$ & Collection \\
\hline Dolichoderus debilis Emery, 1890* & 19 & $\mathrm{Am}$ & Collection \\
\hline Dolichoderus diversus Emery, 1894* & 5,43 & $\mathrm{Am}, \mathrm{Ce}$ & Collection \\
\hline Dolichoderus imitator Emery, $1894^{*}$ & $5,13,31,40,52,57,50$ & $\mathrm{Am}, \mathrm{Ce}$ & Collection \\
\hline Dolichoderus lamellosus (Mayr, 1870) & $3,10,31,45$ & $\mathrm{Am}, \mathrm{Ce}, \mathrm{ACT}$ & Kempf, 1972a, 1972b; Collection \\
\hline Dolichoderus lutosus (Smith, 1858) & $14,57,59$ & $\mathrm{Am}, \mathrm{Ce}$ & Andrade-Silva et al., 2015; Collection \\
\hline Dolichoderus quadridenticulatus (Roger, 1862) & $5,24,45,57,64$ & $\mathrm{Am}, \mathrm{Ce}, \mathrm{ACT}$ & Kempf, 1972a; Collection \\
\hline Dorymyrmex Mayr, 1866 & - & - & - \\
\hline Dorymyrmex biconis Forel, $1912^{*}$ & 52 & $A m$ & Collection \\
\hline Dorymyrmex brunneus Forel, 1908 & $5,13,34,40,52,57,59,61$ & $\mathrm{Am}, \mathrm{Ce}$ & Andrade-Silva et al., 2015; Pereira et al., 2017; Collection \\
\hline Dorymyrmex goeldii Forel, 1904* & 61 & $\mathrm{Am}$ & Collection \\
\hline Dorymyrmex jheringi Forel, 1912 & 11,13 & Ce & Brandão et al., 2011; Collection \\
\hline Dorymyrmex pyramicus (Roger, 1863) & 11 & Ce & Brandão et al., 2011; Collection \\
\hline Dorymyrmex spurius Santschi, 1929 & 11,13 & Ce & Brandão et al., 2011; Collection \\
\hline Dorymyrmex thoracicus Gallardo, 1916 & $4,10,11,13,45,57$ & Am, Ce, ACT & Brandão et al., 2011; Collection \\
\hline Forelius Emery, 1888 & - & - & - \\
\hline Forelius brasiliensis (Forel, 1908) & 11,13 & Ce & Brandão et al., 2011; Collection \\
\hline Forelius maranhaoensis Cuezzo, 2000 & $11,13,35,37,57,62$ & $\mathrm{Am}, \mathrm{Ce}, \mathrm{ACT}$ & Cuezzo, 2000; Brandão et al., 2011; Ulysséa et al., 2017; Collection \\
\hline Forelius pusillus Santschi, 1922* & 13 & Ce & Collection \\
\hline Gracilidris Wild \& Cuezzo, 2006 & - & - & - \\
\hline Gracilidris pombero Wild \& Cuezzo, 2006 & 11,13 & $\mathrm{Ce}$ & Wild \& Cuezzo, 2006; Brandão et al., 2011; Collection \\
\hline Linepithema Mayr, 1866 & - & - & - \\
\hline Linepithema cerradense Wild, 2007 & 11 & Ce & Brandão et al., 2011; Collection \\
\hline Linepithema neotropicum Wild, 2007 & $11,13,45$ & Ce & Wild, 2007; Brandão et al., 2011; Collection \\
\hline Tapinoma Foerster, $1850^{*}$ & - & - & - \\
\hline Tapinoma melanocephalum (Fabricius, 1793)* & $5,34,40,52,57,60$ & $A m$ & Collection \\
\hline Dorylinae Leach, 1815 & - & - & - \\
\hline Acanthostichus Mayr, 1887* & - & - & - \\
\hline Acanthostichus brevicornis Emery, 1894* & 34,52 & Am & Collection \\
\hline Eciton Latreille, 1804 & - & - & - \\
\hline Eciton burchellii (Westwood, 1842)* & $1,21,31,33$ & $\mathrm{Am}, \mathrm{Ce}, \mathrm{ACT}$ & Colletion \\
\hline Eciton mexicanum Roger, 1863* & 60 & Am & Collection \\
\hline Eciton quadriglume (Haliday, 1836) & $1,35,45$ & $\mathrm{Am}, \mathrm{Ce}, \mathrm{ACT}$ & Kempf, 1972a; Watkins, 1976; Collection \\
\hline Eciton rapax Smith, $1855^{*}$ & 3,31 & Am, Ce & Collection \\
\hline Labidus Jurine, 1807 & - & - & - \\
\hline Labidus coecus (Latreille, 1802)* & $5,11,13,14,37,41,52,56,57$ & $\mathrm{Am}, \mathrm{Ce}, \mathrm{ACT}$ & Brandão et al., 2011; Collection \\
\hline Labidus mars (Forel, 1912)* & 34 & $\mathrm{Am}$ & Collection \\
\hline Labidus praedator (Smith, 1858) & 45 & $\mathrm{Ce}$ & Borgmeier, 1955; Watkins, 1976; Kempf, 1972a; Collection \\
\hline Neocerapachys Borowiec, 2016* & - & - & - \\
\hline Neocerapachys splendens (Borgmeier, 1957)* & $5,31,40$ & $\mathrm{Am}, \mathrm{Ce}$ & Collection \\
\hline Nomamyrmex Borgmeier, 1936* & - & - & - \\
\hline Nomamyrmex esenbeckii (Westwood, 1842)* & 23 & Ce & Collection \\
\hline Ectatomminae Emery, 1895 & - & - & - \\
\hline Ectatomma Smith, 1858 & - & - & - \\
\hline
\end{tabular}




\begin{tabular}{|c|c|c|c|}
\hline Taxon name & Locality & Biome & Source \\
\hline Ectatomma brunneum Smith, 1858 & $\begin{array}{l}2,4,5,10,11,12,13,17,19,21,34,35 \\
37,43,48,49,54,56,57,59\end{array}$ & $\mathrm{Am}, \mathrm{Ce}, \mathrm{ACT}$ & $\begin{array}{l}\text { Kempf, 1972a; Brandão et al., 2011; Dáttilo et al., 2012; Andrade-Silva et al., 2015; Pereira } \\
\text { et al., 2017; Collection }\end{array}$ \\
\hline Ectatomma edentatum Roger, 1863 & $11,13,44$ & $\mathrm{Am}, \mathrm{Ce}$ & Brandão et al., 2011; Collection \\
\hline Ectatomma lugens Emery, 1894* & $3,19,34,40$ & $A m$ & Collection \\
\hline Ectatomma muticum Mayr, 1870 & $11,13,22,24,35,45$ & $\mathrm{Ce}, \mathrm{C}$ & Kempf, 1972a; Brandão et al., 2011; Collection \\
\hline Ectatomma opaciventre (Roger, 1861) & 11,13 & $\mathrm{Ce}$ & Brandão et al., 2011; Collection \\
\hline Ectatomma permagnum Forel, $1908^{*}$ & 35,37 & ACT & Collection \\
\hline Ectatomma planidens Borgmeier, 1939 & 11,13 & $\mathrm{Ce}$ & Brandão et al., 2011; Collection \\
\hline Ectatomma ruidum (Roger, 1860)* & 5 & Am & Collection \\
\hline Ectatomma suzanae Almeida Filho, $1986^{*}$ & 37,56 & Am, ACT & Collection \\
\hline Ectatomma tuberculatum (0livier, 1792) & $2,3,5,21,37,40,41,56,57,59$ & $\mathrm{Am}, \mathrm{Ce}, \mathrm{ACT}$ & Andrade-Silva et al., 2015; Collection \\
\hline Gnamptogenys Roger, 1863 & - & - & - \\
\hline Gnamptogenys acuminata (Emery, 1896)* & $31,40,57,58$ & $\mathrm{Am}, \mathrm{Ce}$ & Collection \\
\hline Gnamptogenys ammophila Lattke, 1990 & 11,13 & Ce & Brandão et al., 2011; Collection \\
\hline Gnamptogenys annulata (Mayr, 1887)* & 60 & Am & Collection \\
\hline Gnamptogenys caelata Kempf, 1967 & 34 & $\mathrm{Am}$ & Dias \& Lattke, 2019 Collection \\
\hline Gnamptogenys haenschi (Emery, 1902)* & $5,6,34,40$ & $\mathrm{Am}$ & Collection \\
\hline Gnamptogenys horni (Santschi, 1929)* & $5,37,40,52$ & Am, ACT & Collection \\
\hline Gnamptogenys lanei Kempf, 1960* & 40 & Am & Collection \\
\hline Gnamptogenys mina (Brown, 1956)* & 34,57 & Am & Collection \\
\hline Gnamptogenys minuta (Emery, 1896) & $30,41,52$ & $\mathrm{Am}, \mathrm{Ce}$ & Dias \& Lattke, 2019 Collection \\
\hline Gnamptogenys moelleri (Forel, 1912)* & $30,41,52,57,63$ & $\mathrm{Am}, \mathrm{Ce}, \mathrm{ACT}$ & Collection \\
\hline Gnamptogenys rastrata (Mayr, 1866)* & 30 & Ce & Collection \\
\hline Gnamptogenys striatula Mayr, $1884^{*}$ & $30,32,34,41$ & $\mathrm{Ce}, \mathrm{ACT}$ & Collection \\
\hline Gnamptogenys sulcata (Smith, 1858)* & 34,37 & $\mathrm{Am}, \mathrm{ACT}$ & Collection \\
\hline Gnamptogenys triangularis (Mayr, 1887)* & 40 & Am & Collection \\
\hline Typhlomyrmex Mayr, 1862* & - & - & - \\
\hline Typhlomyrmex rogenhoferi Mayr, 1862* & 1 & Am & Collection \\
\hline Formicinae Latreille, 1809 & - & - & - \\
\hline Acropyga Roger, $1862^{*}$ & - & - & - \\
\hline Acropyga goeldii Forel, 1893* & 57 & Am & Collection \\
\hline Acropyga smithii Forel, 1893* & 4 & $\mathrm{Am}$ & Collection \\
\hline Brachymyrmex Mayr, 1868 & - & - & - \\
\hline Brachymyrmex australis Forel, 1901 & 11,13 & Ce & Brandão et al., 2011; Collection \\
\hline Brachymyrmex heeri Forel, $1874^{*}$ & $5,34,37,40,46,52,56,57$ & Am, ACT & Collection \\
\hline Brachymyrmex patagonicus Mayr, 1868 & 11,13 & $\mathrm{Ce}$ & Brandão et al., 2011; Collection \\
\hline Camponotus Mayr, 1861 & - & - & - \\
\hline Camponotus arboreus (Smith, 1858) & 10,45 & $\mathrm{Ce}, \mathrm{ACT}$ & Mann, 1916; Kempf, 1972a; Collection \\
\hline Camponotus atriceps (Smith, 1858) & $9,29,37,38,53,57$ & $\mathrm{Am}, \mathrm{Ce}, \mathrm{ACT}$ & Dáttilo et al., 2012; Collection \\
\hline Camponotus balzani Emery, 1894* & 37 & AST & Collection \\
\hline Camponotus bidens Mayr, 1870* & $5,31,34$ & $\mathrm{Am}, \mathrm{Ce}$ & Collection \\
\hline Camponotus blandus (Smith, 1858) & $\begin{array}{c}5,11,17,24,28,31,34,35,40,43,56 \\
57,59,65\end{array}$ & $\mathrm{Am}, \mathrm{Ce}, \mathrm{ACT}$ & Brandão et al., 2011; Andrade-Silva et al., 2015; Collection \\
\hline Camponotus cameranoi Emery, $1894^{*}$ & 34 & Am & Collection \\
\hline Camponotus chartifex (Smith, 1860)* & 5 & Am & - \\
\hline Camponotus crassus Mayr, 1862 & $\begin{array}{l}5,10,11,30,31,35,37,40,45,52 \\
56,57\end{array}$ & $\mathrm{Am}, \mathrm{Ce}, \mathrm{ACT}$ & Kempf, 1972a; Brandão et al., 2011; Collection \\
\hline Camponotus fastigatus Roger, $1863^{*}$ & 37,56 & $\mathrm{Am}$ & Collection \\
\hline Camponotus femoratus Fabricius, 1804)* & 4 & Am & Collection \\
\hline Camponotus godmani Forel, 1899* & $5,34,60$ & Am & Collection \\
\hline Camponotus latangulus Roger, $1863^{*}$ & $5,34,40,52,58$ & $A m$ & Collection \\
\hline Camponotus leydigi Forel, 1886 & $5,10,17,40,43,44,45,47,53,56$ & $\mathrm{Am}, \mathrm{Ce}, \mathrm{ACT}$ & Kempf, 1972a; Collection \\
\hline Camponotus melanoticus Emery, 1894 & $37,56,57,59$ & Am, ACT & Andrade-Silva et al., 2015; Collection \\
\hline Camponotus novogranadensis Mayr, $1870^{*}$ & $5,35,37,57$ & Am, ACT & Collection \\
\hline Camponotus personatus Emery, 1894 & 11 & $\mathrm{Ce}$ & Brandão et al., 2011; Collection \\
\hline Camponotus rectangularis Emery, 1890* & 62 & $\mathrm{Am}$ & Collection \\
\hline Camponotus renggeri Emery, 1894 & $11,19,27,37,42,43,45,53,56,64$ & $\mathrm{Am}, \mathrm{Ce}, \mathrm{ACT}$ & Kempf, 1972a; Brandão et al., 2011; Collection \\
\hline Camponotus rufipes (Fabricius, 1775) & 57,59 & $\mathrm{Am}$ & Andrade-Silva et al., 2015; Pereira et al., 2017 \\
\hline Camponotus senex (Smith, 1858) & 57,59 & Am & Andrade-Silva et al., 2015 \\
\hline Camponotus sexguttatus (Fabricius, 1793)* & 58 & $\mathrm{Am}$ & Collection \\
\hline Camponotus silvestrii Emery, 1906 & 35 & ACT & Janicki et al., 2016 \\
\hline Camponotus substitutus Emery, $1894^{*}$ & $4,10,30,41,57$ & $\mathrm{Am}, \mathrm{Ce}, \mathrm{ACT}$ & Collection \\
\hline
\end{tabular}




\begin{tabular}{|c|c|c|c|}
\hline Taxon name & Locality & Biome & Source \\
\hline Camponotus tenuiscapus Roger, $1863^{*}$ & 34 & Am & Collection \\
\hline Camponotus trapeziceps Forel, $1908^{*}$ & $5,34,46$ & Am & Collection \\
\hline Camponotus trapezoideus Mayr, $1870^{*}$ & $5,34,40$ & $\mathrm{Am}$ & Collection \\
\hline Gigantiops Roger, 1863 & - & - & - \\
\hline Gigantiops destructor (Fabricius, 1804) & $12,13,14,45,57$ & $\mathrm{Am}, \mathrm{Ce}$ & Forel, 1904; Wheeler, 1922; Kempf, 1972a; Collection \\
\hline Nylanderia Emery, 1906* & - & - & - \\
\hline Nylanderia fulva (Mayr, 1862)* & $5,10,13,52,57$ & $\mathrm{Am}, \mathrm{Ce}, \mathrm{ACT}$ & Collection \\
\hline Nylanderia guatemalensis (Forel, 1885)* & 34,40 & Am & Collection \\
\hline Paratrechina Motschoulsky, 1863* & - & - & - \\
\hline Paratrechina longicornis (Latreille, 1802)* & $10,13,37,57,58$ & $\mathrm{Am}, \mathrm{Ce}, \mathrm{ACT}$ & Collection \\
\hline Myrmicinae Lepeletier de Saint-Fargeau, 1835 & - & - & - \\
\hline Acromyrmex Mayr, 1865 & - & - & - \\
\hline Acromyrmex hystrix (Latreille, 1802) & 29 & Ce & Dáttilo et al., 2010 \\
\hline Acromyrmex landolti (Forel, 1885) & $11,45,57$ & $\mathrm{Am}, \mathrm{Ce}$ & Gonçalves, 1961; Kempf, 1972a; Brandão et al., 2011; Collection \\
\hline Acromyrmex laticeps (Emery, 1905)* & 22 & $\mathrm{Ce}$ & Collection \\
\hline Acromyrmex nigrosetosus (Forel, 1908) & 22,45 & Ce & Gonçalves, 1961; Kempf, 1972a; Collection \\
\hline Acromyrmex rugosus (Smith, 1858) & $4,11,22,45,57,59$ & $\mathrm{Am}, \mathrm{Ce}$ & Gonçalves, 1961; Brandão et al., 2011; Andrade-Silva et al., 2015; Collection \\
\hline Acromyrmex subterraneus (Forel, 1893)* & 40 & Am & Collection \\
\hline Apterostigma Mayr, $1865^{*}$ & - & - & - \\
\hline Apterostigma robustum Emery, $1896^{*}$ & $34,40,52$ & $\mathrm{Am}$ & Collection \\
\hline Atta Fabricius, 1804 & - & - & - \\
\hline Atta cephalotes (Linnaeus, 1758) & 45 & $\mathrm{Ce}$ & Kempf, 1972a; Collection \\
\hline Atta laevigata (Smith, 1858) & 45 & Ce & Kempf, 1972a; Collection \\
\hline Atta opaciceps Borgmeier, 1939* & $35,37,63$ & Am, ACT & Collection \\
\hline Atta sexdens (Linnaeus, 1758) & $11,31,45,57$ & $\mathrm{Am}, \mathrm{Ce}$ & Gonçalves, 1942, 1947; Kempf, 1972a; Brandão et al., 2011; Collection \\
\hline Basiceros Schulz, 1906 & - & - & - \\
\hline Basiceros militaris (Weber, 1950) & $4,34,55$ & Am & Janicki et al., 2016; Collection \\
\hline Basiceros scambognathus (Brown, 1949) & 30 & Ce & Feitosa et al., 2007; Collection \\
\hline Blepharidatta Wheeler, 1915 & - & - & - \\
\hline Blepharidatta conops Kempf, 1967 & $11,13,30,32$ & $\mathrm{Ce}$ & Silva, 2007; Brandão et al., 2011; Pereira et al., 2014; Brandão et al., 2015; Collection \\
\hline Cardiocondyla Emery, 1869* & - & - & - \\
\hline Cardiocondyla emeryi Forel, $1881^{*}$ & 10,13 & $\mathrm{Ce}, \mathrm{ACT}$ & Collection \\
\hline Cardiocondyla obscurior Wheeler, $1929^{*}$ & 52 & Am & Collection \\
\hline Carebara Westwood, $1840^{*}$ & - & - & - \\
\hline Carebara arabara Fernández, 2010* & 4 & Am & Collection \\
\hline Carebara brevipilosa Fernández, 2004* & 4 & Am & Collection \\
\hline Carebara urichi (Wheeler, 1922)* & 34,40 & Am & Collection \\
\hline Cephalotes Latreille, 1802 & - & - & - \\
\hline Cephalotes atratus (Linnaeus, 1758) & $3,5,9,13,17,21,27,33,51,52,57,60$ & $\mathrm{Am}, \mathrm{Ce}, \mathrm{ACT}$ & De Andrade \& Baroni Urbani, 1999; Brandão et al., 2011; Collection \\
\hline Cephalotes clypeatus (Fabricius, 1804) & $5,3,30$ & $\mathrm{Am}, \mathrm{Ce}$ & De Andrade \& Baroni Urbani, 1999; Collection \\
\hline Cephalotes cordatus (Smith, 1853) & 45,57 & $\mathrm{Am}, \mathrm{Ce}$ & Kempf, 1972a; Kempf, 1960a; Brandão, 1991; Collection \\
\hline Cephalotes eduarduli (Forel, 1921)* & 10 & ACT & Collection \\
\hline Cephalotes grandinosus (Smith, 1860)* & 63 & Am & Collection \\
\hline Cephalotes maculatus (Smith, 1876)* & 5,57 & Am & Collection \\
\hline Cephalotes marginatus (Fabricius, 1804) & 38 & Ce & De Andrade \& Baroni Urbani, 1999; Collection \\
\hline Cephalotes minutus (Fabricius, 1804) & $5,38,40,41,52,61$ & $\mathrm{Am}, \mathrm{Ce}, \mathrm{ACT}$ & De Andrade \& Baroni Urbani, 1999; Collection \\
\hline Cephalotes pavonii (Latreille, 1809) & $11,13,45,52$ & $\mathrm{Am}, \mathrm{Ce}$ & Kempf, 1972a; Kempf, 1960a; Brandão, 1991; Collection \\
\hline Cephalotes pilosus (Emery, 1896)* & $35,43,64$ & $\mathrm{Ce}, \mathrm{ACT}$ & Collection \\
\hline Cephalotes pusillus (Klug, 1824) & $\begin{array}{c}1,10,11,13,17,29,31,34,35,37,43 \\
45,57,61,64\end{array}$ & $\mathrm{Am}, \mathrm{Ce}, \mathrm{ACT}$ & Kempf, 1972a; Kempf, 1960a; Brandão, 1991; De Andrade \& Baroni Urbani, 1999; Collection \\
\hline Cephalotes serraticeps (Smith, 1858) & 9 & $\mathrm{Am}$ & De Andrade \& Baroni Urbani, 1999; Collection \\
\hline Cephalotes umbraculatus (Fabricius, 1804)* & 57 & $A m$ & Collection \\
\hline Crematogaster Lund, 1831 & - & - & - \\
\hline Crematogaster abstinens Forel, 1899* & 13,56 & $\mathrm{Am}, \mathrm{Ce}$ & Collection \\
\hline Crematogaster acuta (Fabricius, 1804)* & 13 & $\mathrm{Ce}$ & Collection \\
\hline Crematogaster brasiliensis Mayr, 1878* & 63 & $\mathrm{Am}$ & Collection \\
\hline Crematogaster curvispinosa Mayr, 1862* & 10 & ACT & Collection \\
\hline Crematogaster erecta Mayr, 1866 & $5,13,34,40,45,52,57$ & $\mathrm{Am}, \mathrm{Ce}$ & Kempf, 1968; Kempf, 1972a; Collection \\
\hline Crematogaster evallans Forel, $1907^{*}$ & 13,57 & $\mathrm{Am}, \mathrm{Ce}$ & Collection \\
\hline Crematogaster limata Smith, $1858^{*}$ & $34,35,37,40,52,57,63$ & Am, ACT & Collection \\
\hline Crematogaster tenuicula Forel, 1904* & $5,34,35,37,52,57,60$ & $\mathrm{Am}, \mathrm{ACT}$ & Collection \\
\hline Crematogaster victima Smith, 1858 & 57,59 & $\mathrm{Am}$ & Andrade-Silva et al. 2015; Collection \\
\hline
\end{tabular}




\begin{tabular}{|c|c|c|c|}
\hline Taxon name & Locality & Biome & Source \\
\hline Cyphomyrmex Mayr, 1862* & - & - & - \\
\hline Cyphomyrmex laevigatus Weber, $1938^{*}$ & 4,40 & Am & Collection \\
\hline Cyphomyrmex major Forel, 1901* & 5,40 & $\mathrm{Am}$ & Collection \\
\hline Cyphomyrmex minutus Mayr, 1862* & 30,31 & Ce & Collection \\
\hline Cyphomyrmex peltatus Kempf, 1966* & $4,5,30,34,37,40,57,60$ & $\mathrm{Am}, \mathrm{Ce}, \mathrm{ACT}$ & Collection \\
\hline Cyphomyrmex transversus Emery, 1894* & $5,37,40,62$ & Am, ACT & Collection \\
\hline Daceton Perty, 1833* & - & - & - \\
\hline Daceton armigerum (Latreille, 1802)* & 4,19 & Am & Collection \\
\hline Hylomyrma Forel, 1912* & - & - & - \\
\hline Hylomyrma balzani (Emery, 1894)* & $34,37,40,57$ & Am, ACT & Collection \\
\hline Hylomyrma immanis Kempf, 1973* & 4 & Am & Collection \\
\hline Hylomyrma longiscapa Kempf, $1961^{*}$ & 4 & Am & Collection \\
\hline Hylomyrma praepotens Kempf, 1973* & 55 & Am & Collection \\
\hline Hylomyrma reginae Kutter, $1977^{*}$ & 55 & Am & Collection \\
\hline Megalomyrmex Forel, 1885* & - & - & - \\
\hline Megalomyrmex drifti Kempf, 1961* & 60 & Am & Collection \\
\hline Monomorium Mayr, 1855* & - & - & - \\
\hline Monomorium floricola (Jerdon, 1851)* & $5,40,43,52$ & $\mathrm{Am}, \mathrm{Ce}$ & Collection \\
\hline Monomorium pharaonis (Linnaeus, 1758)* & 37,57 & $\mathrm{Am}, \mathrm{ACT}$ & Collection \\
\hline Mycetarotes Emery, 1913* & - & - & - \\
\hline Mycetarotes parallelus (Emery, 1906)* & 57 & $\mathrm{Am}$ & Collection \\
\hline Mycocepurus Forel, 1893 & - & - & - \\
\hline Mycocepurus goeldii (Forel, 1893) & $11,37,52,56,57$ & $\mathrm{Am}, \mathrm{Ce}, \mathrm{ACT}$ & Brandão et al., 2011; Collection \\
\hline Mycocepurus smithii (Forel, 1893)* & 4,63 & Am & Collection \\
\hline Nesomyrmex Wheeler, 1910 & - & - & - \\
\hline Nesomyrmex asper (Mayr, 1887)* & 52 & $\mathrm{Am}$ & Collection \\
\hline Nesomyrmex brasiliensis (Kempf, 1958) & 11,13 & Ce & Brandão et al., 2011; Collection \\
\hline Nesomyrmex spininodis (Mayr, 1887)* & 5,52 & Am & Collection \\
\hline Nesomyrmex wilda (Smith, 1943)* & 60 & Am & Collection \\
\hline Ochetomyrmex Mayr, 1878 & - & - & - \\
\hline Ochetomyrmex neopolitus Fernández, 2003* & 4,37 & Am, ACT & Collection \\
\hline Ochetomyrmex semipolitus Mayr, 1878 & $11,12,13,45$ & $\mathrm{Ce}$ & Brandão et al., 2011; Collection \\
\hline Octostruma Forel, 1912 & - & - & - \\
\hline Octostruma balzani (Emery, 1894) & $30,45,60$ & $\mathrm{Am}, \mathrm{Ce}$ & Janicki et al., 2016; Collection \\
\hline Octostruma iheringi (Emery, 1888)* & 34,40 & Am & Collection \\
\hline Oxyepoecus Santschi, 1926* & - & - & - \\
\hline Oxyepoecus vezenyii (Forel, 1907)* & 15 & Ce & Collection \\
\hline Pheidole Westwood, 1839 & - & - & - \\
\hline Pheidole allarmata Wilson, 2003* & 34,52 & $\mathrm{Am}$ & Collection \\
\hline Pheidole diligens (Smith, 1858)* & 37 & ACT & Collection \\
\hline Pheidole dolon Wilson, 2003** & 34 & Am & Collection \\
\hline Pheidole fallax Mayr, $1870^{*}$ & 5,37 & Am, ACT & Collection \\
\hline Pheidole fimbriata Roger, $1863^{*}$ & 4 & Am & Collection \\
\hline Pheidole flavens Roger, 1863 & 45 & $\mathrm{Ce}$ & Janicki et al., 2016; Collection \\
\hline Pheidole fracticeps Wilson, 2003* & 31 & $\mathrm{Ce}$ & Collection \\
\hline Pheidole gauthieri Forel, 1901* & 34 & Am & Collection \\
\hline Pheidole impressa Mayr, $1870^{*}$ & 10,57 & Am, ACT & Collection \\
\hline Pheidole jeannei Wilson, 2003* & 37 & $\mathrm{ACT}$ & Collection \\
\hline Pheidole microps Wilson, $2003^{* *}$ & 41 & $A m$ & Collection \\
\hline Pheidole midas Wilson, 2003* & 41,52 & Am & Collection \\
\hline Pheidole obscurithorax Naves, 1985 & 29 & ACT & Dáttilo et al., 2012 \\
\hline Pheidole radoszkowskii Mayr, 1884 & $5,34,35,37,40,52,56,57,59$ & Am, ACT & Andrade-Silva et al., 2015; Collection \\
\hline Pheidole scolioceps Wilson, 2003* & 4,37 & Am, ACT & Collection \\
\hline Pheidole sensitiva Borgmeier, 1959** & 30 & Ce & Collection \\
\hline Pheidole susannae Forel, 1886* & 37 & ACT & Collection \\
\hline Pheidole synarmata Wilson, 2003 & 57,59 & $\mathrm{Am}$ & Andrade-Silva et al., 2015; Pereira et al., 2017; Collection \\
\hline Pheidole transversostriata Mayr, 1887* & 37 & ACT & Collection \\
\hline Pogonomyrmex Mayr, 1868 & - & - & - \\
\hline Pogonomyrmex naegelii Emery, 1878 & 14 & $\mathrm{Ce}$ & Johnson, 2015 \\
\hline Procryptocerus Emery, 1887 & - & - & - \\
\hline Procryptocerus goeldii Forel, 1899 & 45 & Sa & Kempf, 1972a \\
\hline Procryptocerus hylaeus Kempf, 1951 & 10,45 & $\mathrm{Ce}, \mathrm{ACT}$ & Longino \& Snelling, 2002; Collection \\
\hline
\end{tabular}




\begin{tabular}{|c|c|c|c|}
\hline Taxon name & Locality & Biome & Source \\
\hline Procryptocerus pictipes Emery, $1896^{*}$ & 5,40 & $\mathrm{Am}$ & Collection \\
\hline Rogeria Emery, 1894 & - & - & - \\
\hline Rogeria alzatei Kugler, 1994* & $5,34,52,57$ & Am & Collection \\
\hline Rogeria besucheti Kugler, 1994* & 40 & $\mathrm{Am}$ & Collection \\
\hline Rogeria germaini Emery, $1894^{*}$ & 40 & $\mathrm{Am}$ & Collection \\
\hline Rogeria lirata Kugler, 1994* & 58 & $\mathrm{Am}$ & Collection \\
\hline Rogeria scobinata Kugler, 1994 & 11 & Ce & Brandão et al., 2011; Collection \\
\hline Sericomyrmex Mayr, 1865 & - & - & - \\
\hline Sericomyrmex mayri Forel, 1912 & $18,29,35,37,45$ & $\mathrm{Am}, \mathrm{Ce}, \mathrm{ACT}$ & Jesovnik \& Schultz, 2017; Collection \\
\hline Sericomyrmex parvulus Forel, 1912 & $18,25,45$ & $\mathrm{Am}, \mathrm{Ce}$ & Jesovnik \& Schultz, 2017; Collection \\
\hline Sericomyrmex saussurei Emery, 1894 & $18,29,45$ & $\mathrm{Am}, \mathrm{Ce}$ & Jesovnik \& Schultz, 2017; Collection \\
\hline Solenopsis Westwood, 1840 & - & - & - \\
\hline Solenopsis geminata (Fabricius, 1804) & $5,34,37,52,57,58$ & Am, ACT & Wauters et al., 2018; Collection \\
\hline Solenopsis globularia (Smith, 1858) & $5,45,52,57,59,61$ & $\mathrm{Am}, \mathrm{Ce}$ & Kempf, 1972a; Andrade-Silva et al., 2015; Collection \\
\hline Solenopsis pollux Forel, 1893* & 37 & ACT & Collection \\
\hline Solenopsis saevissima (Smith, 1855) & $37,57,59,61,65$ & Am, ACT & Shoemaker et al., 2006; Andrade-Silva et al., 2015; Pereira et al., 2017; Collection \\
\hline Solenopsis substituta Santschi, $1925^{*}$ & 57,61 & Am & Collection \\
\hline Solenopsis virulens (Smith, 1858)* & $5,34,40,52$ & Am & Collection \\
\hline Stegomyrmex Emery, 1912 & - & - & - \\
\hline Stegomyrmex olindae Feitosa, Brandão \& Diniz, 2008 & $2,29,45$ & $\mathrm{Am}, \mathrm{Ce}$ & Feitosa et al., 2008; Ulysséa et al., 2015; Collection \\
\hline Strumigenys Smith, $1860^{*}$ & - & - & - \\
\hline Strumigenys alberti Forel, 1893* & 4 & Am & Collection \\
\hline Strumigenys crassicornis Mayr, $1887^{*}$ & $30,32,55$ & $\mathrm{Am}, \mathrm{Ce}$ & Collection \\
\hline Strumigenys denticulata Mayr, $1887^{*}$ & $5,13,30,31,34,40,52,55$ & $\mathrm{Am}, \mathrm{Ce}$ & Collection \\
\hline Strumigenys eggersi Emery, 1890* & $4,30,31,32,41,60$ & $\mathrm{Am}, \mathrm{Ce}, \mathrm{ACT}$ & Collection \\
\hline Strumigenys elongata Roger, $1863^{*}$ & $4,13,30,31,41$ & Am, Ce & Collection \\
\hline Strumigenys gytha Bolton, 2000* & 41 & $\mathrm{Am}$ & Collection \\
\hline Strumigenys hyphata (Brown, 1953)* & 4,30 & $\mathrm{Am}, \mathrm{Ce}$ & Collection \\
\hline Strumigenys metopia (Brown, 1959)* & 4 & $\mathrm{Am}$ & Collection \\
\hline Strumigenys mirabilis Mann, 1926* & 4 & $\mathrm{Am}$ & Collection \\
\hline Strumigenys orchibia (Brown, 1953) ${ }^{* *}$ & 60 & $A m$ & Collection \\
\hline Strumigenys schmalzi Emery, 1906* & 30,31 & Ce & Collection \\
\hline Strumigenys subedentata Mayr, $1887^{*}$ & $30,31,40$ & $\mathrm{Am}, \mathrm{Ce}$ & Collection \\
\hline Strumigenys trudifera Kempf \& Brown, 1969* & $4,5,40$ & Am & Collection \\
\hline Strumigenys urrhobia (Bolton, 2000)* & 56 & $\mathrm{Am}$ & Collection \\
\hline Strumigenys villiersi (Perrault, 1986)* & 30 & Ce & Collection \\
\hline Strumigenys zeteki (Brown, 1959)* & 12 & Ce & Collection \\
\hline Tetramorium Mayr, 1855* & - & - & - \\
\hline Tetramorium simillimum (Smith, 1851) & 10 & ACT & Kempf, 1972a, 1975; Brandão, 1991; Collection \\
\hline Trachymyrmex Forel, 1893 & - & - & - \\
\hline Trachymyrmex bugnioni (Forel, 1912) & 11,30 & Ce & Brandão et al., 2011; Collection \\
\hline Trachymyrmex relictus Borgmeier, 1934* & $34,35,37,40,52,57,61$ & Am & Collection \\
\hline Tranopelta Mayr, 1866* & & - & - \\
\hline Tranopelta gilva Mayr, 1866* & 5,57 & $\mathrm{Am}$ & Collection \\
\hline Wasmannia Forel, 1893 & - & - & - \\
\hline Wasmannia auropunctata (Roger, 1863) & $\begin{array}{c}5,11,12,13,15,30,31,32,34,35,37 \\
40,52,55,57,61\end{array}$ & $\mathrm{Am}, \mathrm{Ce}, \mathrm{ACT}$ & Brandão et al., 2011; Collection \\
\hline Paraponerinae Emery, 1901 & - & - & - \\
\hline Paraponera Smith, 1858 & - & - & - \\
\hline Paraponera clavata (Fabricius, 1775) & $2,3,7,19,36,38,42,45,50$ & $\mathrm{Am}, \mathrm{Ce}, \mathrm{ACT}$ & Ward \& Downie, 2005; Ward, 2007; Collection \\
\hline Ponerinae Lepeletier de Saint-Fargeau, 1835 & - & - & - \\
\hline Anochetus Mayr, $1861^{*}$ & - & - & - \\
\hline Anochetus bispinosus (Smith, 1858)* & 5,34 & $A m$ & Collection \\
\hline Anochetus diegensis Forel, 1912* & $5,34,40,57,60$ & Am & Collection \\
\hline Anochetus horridus Kempf, 1964* & $4,27,37$ & $\mathrm{Am}, \mathrm{Ce}, \mathrm{ACT}$ & Collection \\
\hline Anochetus mayri Emery, 1884* & $5,34,52,57$ & $\mathrm{Am}$ & Collection \\
\hline Centromyrmex Mayr, 1866* & - & - & - \\
\hline Centromyrmex brachycola (Roger, 1861)* & $5,34,57,61$ & $\mathrm{Am}$ & Collection \\
\hline Cryptopone Emery, 1893* & - & - & - \\
\hline Cryptopone guianensis (Weber, 1939)* & 34 & $\mathrm{Am}$ & Collection \\
\hline Dinoponera Roger, 1861 & - & & - \\
\hline
\end{tabular}




\begin{tabular}{|c|c|c|c|}
\hline Taxon name & Locality & Biome & Source \\
\hline Dinoponera gigantea (Perty, 1833) & $1,11,13,33,45$ & $\mathrm{Am}, \mathrm{Ce}, \mathrm{ACT}$ & Kempf, 1971, 1972a; Monnin et al., 2003; Brandão et al., 2011; Collection \\
\hline Hypoponera Santschi, 1938* & - & - & - \\
\hline Hypoponera distinguenda (Emery, 1890)* & 34 & Am & Collection \\
\hline Hypoponera opacior (Forel, 1893)* & 61 & $A m$ & Collection \\
\hline Hypoponera trigona (Mayr, 1887)* & $5,34,52,57$ & Am & Collection \\
\hline Leptogenys Roger, 1861 & & - & - \\
\hline Leptogenys guianensis Wheeler, $1923^{*}$ & 57 & $A m$ & Collection \\
\hline Leptogenys unistimulosa Roger, 1863 & 30,63 & $\mathrm{Am}, \mathrm{Ce}$ & Lattke, 2011; Collection \\
\hline Mayaponera Schmidt \& Shattuck, 2014* & - & - & - \\
\hline Mayaponera constricta (Mayr, 1884)* & $5,30,35,37,40,60$ & $\mathrm{Am}, \mathrm{Ce}, \mathrm{ACT}$ & Collection \\
\hline Neoponera Emery, 1901 & - & - & - \\
\hline Neoponera commutata (Roger, 1860) & $7,21,45,56$ & $\mathrm{Am}, \mathrm{Ce}$ & Kempf, 1959; Kempf, 1972a; Collection \\
\hline Neoponera marginata (Roger, 1861)* & 57 & Am & Collection \\
\hline Neoponera striatinodis (Emery, 1890)* & 5 & $\mathrm{Am}$ & Collection \\
\hline Neoponera unidentata (Mayr, 1862)* & 34 & $\mathrm{Am}$ & Collection \\
\hline Neoponera verenae Forel, $1922^{*}$ & 34,37 & Am, ACT & Collection \\
\hline Neoponera villosa (Fabricius, 1804) & $11,13,19,60$ & $\mathrm{Am}, \mathrm{Ce}$ & Brandão et al., 2011; Fernandes et al., 2014; Collection \\
\hline Odontomachus Latreille, 1804 & - & - & - \\
\hline Odontomachus bauri Emery, $1892^{*}$ & $11,22,35,37,57,59,60$ & $\mathrm{Am}, \mathrm{Ce}, \mathrm{ACT}$ & Brandão et al., 2011; Andrade-Silva et al., 2015; Collection \\
\hline Odontomachus brunneus (Patton, 1894)* & 22 & Ce & Collection \\
\hline Odontomachus chelifer (Latreille, 1802)* & 57,26 & $\mathrm{Am}, \mathrm{Ce}$ & Collection \\
\hline Odontomachus haematodus (Linnaeus, 1758) & $1,45,57$ & $\mathrm{Am}, \mathrm{Ce}$ & Janicki et al., 2016; Collection \\
\hline Odontomachus meinerti Forel, 1905* & 34,52 & $\mathrm{Am}$ & Collection \\
\hline Odontomachus opaciventris Forel, 1899 & 29 & ACT & Dáttilo et al. 2012 \\
\hline Odontomachus scalptus Brown, 1978* & 5 & $\mathrm{Am}$ & Collection \\
\hline Pachycondyla Smith, 1858* & - & - & - \\
\hline Pachycondyla crassinoda (Latreille, 1802)* & $5,30,32,35,37,52,57,60$ & $\mathrm{Am}, \mathrm{Ce}, \mathrm{ACT}$ & Collection \\
\hline Pachycondyla harpax (Fabricius, 1804)* & $\begin{array}{c}5,30,31,32,34,35,37,40,46,52 \\
55,56,57\end{array}$ & $\mathrm{Am}, \mathrm{Ce}, \mathrm{ACT}$ & Collection \\
\hline Pachycondyla impressa (Roger, 1861)* & 5 & $A m$ & Collection \\
\hline Pachycondyla lenis Kempf, $1961^{*}$ & 34 & $\mathrm{Am}$ & Collection \\
\hline Platythyrea Roger, 1863 & - & - & - \\
\hline Platythyrea angusta Forel, 1901 & 45 & $\mathrm{Ce}$ & Forel, 1904; Kempf, 1964, 1972a; Collection \\
\hline Platythyrea pilosula (Smith, 1858)* & 60 & Am & Collection \\
\hline Pseudoponera Emery, 1900* & & - & - \\
\hline Pseudoponera gilberti (Kempf, 1960)* & $5,34,40,52,61$ & $\mathrm{Am}$ & Collection \\
\hline Pseudoponera stigma (Fabricius, 1804)* & 41 & $\mathrm{Am}$ & Collection \\
\hline Rasopone Schmidt \& Shattuck, 2014* & - & - & - \\
\hline Rasopone arhuaca (Forel, 1901)* & $5,34,41,52,55,57$ & Am, ACT & Collection \\
\hline Rasopone ferruginea (Smith, 1858)* & $5,30,31,34,40,55$ & $\mathrm{Am}, \mathrm{Ce}$ & Collection \\
\hline Proceratiinae Emery, 1895* & - & - & - \\
\hline Discothyrea Roger, 1863* & - & - & - \\
\hline Discothyrea sexarticulata Borgmeier, $1954^{*}$ & 4,5 & $\mathrm{Am}$ & Collection \\
\hline Pseudomyrmecinae Smith, 1952 & - & - & - \\
\hline Pseudomyrmex Lund, 1831 & - & - & - \\
\hline Pseudomyrmex curacaensis (Forel, 1912) & $5,10,40,45,52$ & $\mathrm{Am}, \mathrm{Ce}, \mathrm{ACT}$ & Ward, 1989; Brandão, 1991; Collection \\
\hline Pseudomyrmex elongatus (Mayr, 1870) & 10,45 & Ce, ACT & Kempf, 1972a; Ward, 1989; Collection \\
\hline Pseudomyrmex ethicus (Forel, 1911)* & 4 & $\mathrm{Am}$ & Collection \\
\hline Pseudomyrmex filiformis (Fabricius, 1804)* & 5,34 & $\mathrm{Am}$ & Collection \\
\hline Pseudomyrmex flavidulus (Smith, 1858) & 11,13 & $\mathrm{Ce}$ & Brandão et al., 2011; Collection \\
\hline Pseudomyrmex gracilis (Fabricius, 1804) & $5,16,38,40,45,46,56,57,63$ & $\mathrm{Am}, \mathrm{Ce}, \mathrm{CCT}$ & Janicki et al., 2016; Collection \\
\hline Pseudomyrmex kuenckeli (Emery, 1890) & 35,43 & Am, ACT & Ward, 1999; Collection \\
\hline Pseudomyrmex oculatus (Smith, 1855) & $5,34,40,45,56,57,63$ & $\mathrm{Am}, \mathrm{Ce}$ & Kempf, 1972a; Collection \\
\hline Pseudomyrmex penetrator (Smith, 1877)* & 3 & $\mathrm{Am}$ & Collection \\
\hline Pseudomyrmex pupa (Forel, 1911)* & 56,40 & $\mathrm{Am}$ & Collection \\
\hline Pseudomyrmex schuppi (Forel, 1901) & $52,57,59$ & $\mathrm{Am}$ & Andrade-Silva et al., 2015; Collection \\
\hline Pseudomyrmex sericeus (Mayr, 1870)* & 5,40 & Am & Collection \\
\hline Pseudomyrmex tenuis (Fabricius, 1804) & $\begin{array}{c}5,13,20,27,34,37,40,45,47,56,58 \\
60,61,62,63\end{array}$ & $\mathrm{Am}, \mathrm{Ce}, \mathrm{ACT}$ & Forel, 1904; Kempf, 1960b, 1972a; Ward \& Downie, 2005; Collection \\
\hline Pseudomyrmex tenuissimus (Emery, 1906) & $5,10,45$ & $\mathrm{Am}, \mathrm{Ce}, \mathrm{ACT}$ & Mann, 1916; Kempf, 1972a; Ward, 1989; Brandão, 1991; Collection \\
\hline Pseudomyrmex termitarius (Smith, 1855) & $5,13,27,43,45,47$ & Am, Ce & Kempf, 1972a; Brandão et al., 2011; Collection \\
\hline Pseudomyrmex urbanus (Smith, 1877) & 13,45 & Ce & Ward, 1989; Brandão, 1991; Collection \\
\hline
\end{tabular}


to identify the morphospecies in ant collections. For instance, 73 ant morphospecies, belonging to 31 ant genera and two subfamilies were here identified at the specific level for the first time (Table 4).

In our data compilation, we found a number of species that were recorded for the first time in the state of Maranhão, but are widely distributed in Brazil (Janicki et al., 2016), as is the case of Dolichoderus imitator Emery, 1894 and Gnamptogenys striatula Mayr, 1884, among others (Table 4). On the other hand, some hyperdiverse and taxonomically challenging genera, such as Pheidole, had a considerable increase in the number of new records. Of the 19 species of Pheidole known to the state, 12 were recorded for the first time in the state of Maranhão, and three species were recorded for the first time in Brazil.

Not surprisingly, the data obtained from the ant literature clearly indicates that taxonomy is the discipline that most contributed to the knowledge of the ant fauna in the state. This is especially true for taxonomic revisions, which deal with large numbers of specimens (e.g., De Andrade \& Baroni Urbani, 1999; Lattke, 2011). The high number of taxonomic publications in our survey is justified by the fact that this discipline was the first area of myrmecology to be developed in Brazil, allowing the formation of large repositories. However, although taxonomy is the discipline with the greatest number of published studies in relation to other areas, in the last 20 years the potential of ant fauna data has been explored in different study areas (Table 2).

Other factors that have contributed to increasing our knowledge of the ant fauna in the state of Maranhão are online tools, which provide high definition images of species (AntWeb, 2019), taxonomic literature (Bolton, 2019), geographic distribution of ant specimens (Janicki et al., 2016), and general information on ant taxa (AntWiki, 2019). These tools facilitate the identification of specimens and provide a fast and effective access to information. In addition, the improvement and development of collection methodologies (Figueiredo et al., 2013) has made the sampling more efficient.

Despite the increased understanding of biodiversity in this region, sampling coverage of ant fauna in Maranhão is strongly irregular (Fig. 1). Our study showed that the Amazon is the better sampled biome and also houses the largest number of species recorded in the state (Table 4). Most collection points are concentrated in the northern region of the state (Fig. 1), which corresponds to the Coastal region of Maranhão, with the highest population density (Chaves et al., 2016), and where the main research centers are located.

While the Cerrado, which corresponds to the biome with the highest coverage in the state (64\%) (MMA, 2011; Stella, 2011), remains poorly sampled with extremely sparse collections (Fig. 1). In relation to this biome, it is in the southern part of the state where most of the collection points are concentrated, which in most cases came from samples derived from environmental impact assessment programs (e.g., Brandão et al., 2011).

The Amazon-Cerrado transition regions are also undersampled in the state, with few records available from taxonomic papers (Kempf, 1972a; Brandão, 1991) and collections. If we want to understand the association between species and forest formations it is essential to characterize species diversity in ecotones, as already observed by other groups (Santos et al., 2010; MaracahipesSantos et al., 2018).

The Caatinga biome remains largely unknown in Maranhão, represented in our study by a single record in the Cerrado-Caatinga transition region (Fig. 1). Although the biome presents a small and fragmented spatial coverage ( $1 \%$ of the state territorial area) (Stella, 2011), the scarcity of information about the ant fauna in the Caatinga has also been observed in other regions of Brazil (Santos et al., 1999; Ulysséa \& Brandão, 2013; Leal et al., 2017). This result illustrates the need for greater collection effort to understand and preserve biodiversity in the Caatinga and, consequently, in the state of Maranhão.

One of the main limitations of the data available to date on the ant fauna in Maranhão was a strong sampling bias, with most samples being collected near the main roads (Fig. 2). This pattern of biased sampling near highways, rivers, coasts, and cities has been reported in several taxonomic groups (Hijmans et al., 2000; Kadmon et al., 2003; Reddy \& Dávalos, 2003; Newbold, 2010; Santos \& Hoppe, 2018), which is explained by the ease access, researchers' interest in certain areas or taxa, and limited financial resources. However, further studies are required to reduce this sampling bias by using different collection methodologies and accessing previously unexplored sites.

Low levels of sampling in conservation areas of the state were also observed (Fig. 3). Conservation areas (i.e., national parks, ecological stations, extractive reserves,

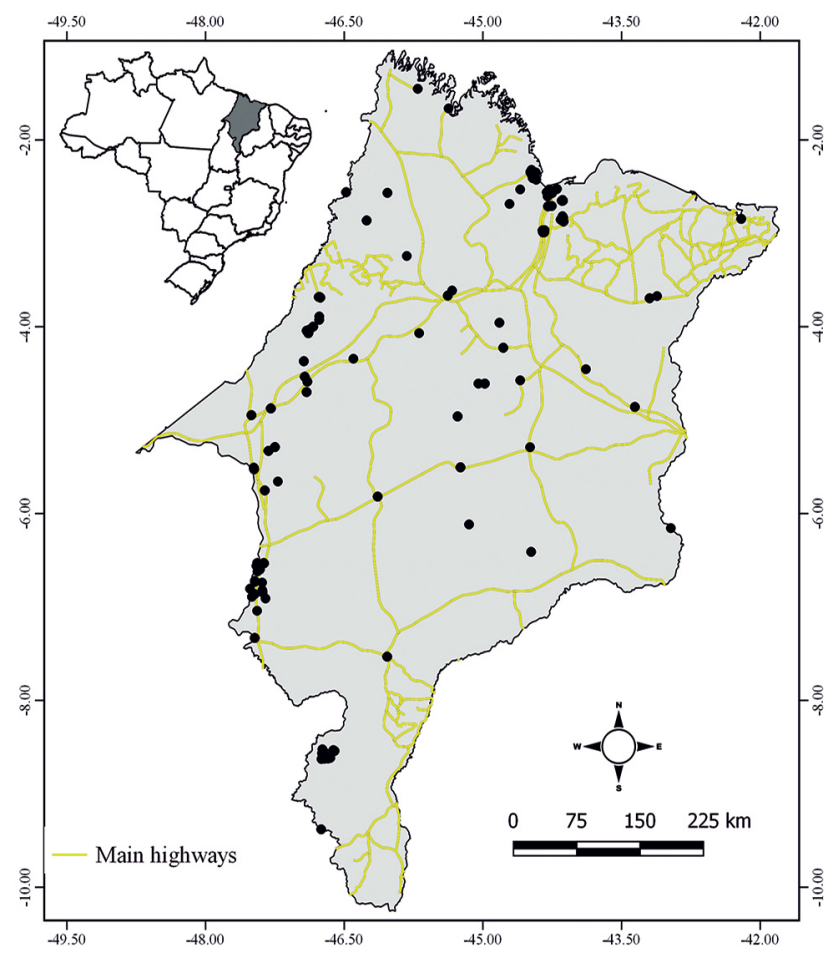

Figure 2. Map of the state of Maranhão emphasizing the main highways and sampling sites of ant species within the state. 


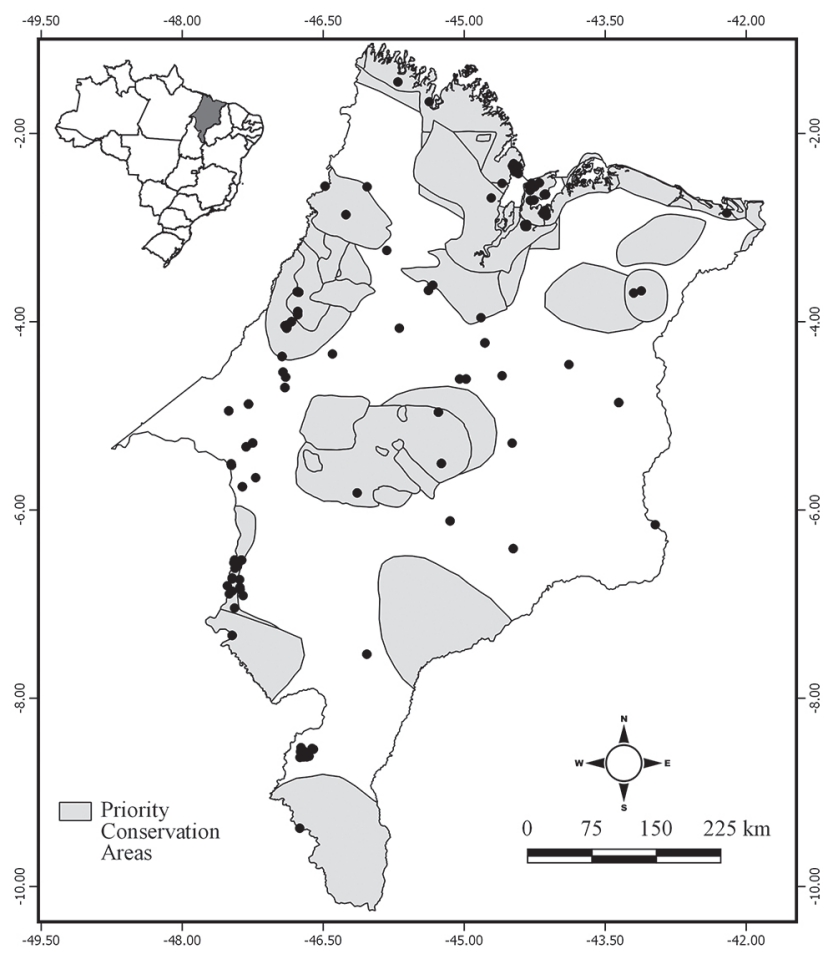

Figure 3. Map of the state of Maranhão emphasizing the Priority Conservation Areas and sampling sites of ant species within the state.

national forests, biological reserves, among others) are of fundamental importance for biodiversity conservation (Peres, 2005) and preserving ecosystem (Hallmann et al., 2017).

To the best of our knowledge, this is the first compilation focused on studying the ant fauna of Maranhão, one of the largest geopolitical regions of Brazil. Our study significantly increase the number of ant species recorded in the state and demonstrates the importance of carrying out planned inventories for a more detailed understanding of the regional ant fauna. Finally, our data provide the baseline information to further explore the ant fauna in Maranhão, to improve current knowledge and to accurately determine the occurrence of several species.

\section{CONCLUSION}

This paper represents an updated record of the ant species occurring in the state of Maranhão, with numbers increasing from 99 to 279 species. Further collection efforts in different biomes are essential for a better understanding of the biodiversity of the state, and for planning long-term conservation action. Ongoing studies on taxonomy, natural history, and ecology are certainly expected to contribute to this.

\section{ACKNOWLEDGMENTS}

We thank the researchers Emília Z. Albuquerque (Arizona State University), Fabrício Baccaro (Universidade Federal do Amazonas), Jacques Delabie (Comissão Executiva do Plano da Lavoura Cacaueira), Mônica
Ulysséa (Museu de Zoologia da Universidade de São Paulo), Phil Ward (University of California) and, Ricardo Vicente (Universidade do Estado de Mato Grosso) for their help with the identification/confirmation of the ant species. ACF and RMF were financed by the Conselho Nacional de Desenvolvimento Científico e Tecnológico (CNPq) (processes 140260/2016-1 and 1302462/2016-3, respectively). LPP, JAS and SPT were financed by the Coordenação de Aperfeiçoamento de Pessoal de Nível Superior - Brasil (CAPES) - Finance Code 001. TSRS was financed by CAPES (process 40001016005P5). JAMG and GXR were financed by the Fundação de Amparo à Pesquisa e ao Desenvolvimento Científico e Tecnológico do Maranhão (FAPEMA) - Finance Code 03135/13.

\section{REFERENCES}

Andrade-Silva, J.; Pereira, E.K.C.; Silva, 0.; Santos, C.L.C.; Delabie, J.H.C. \& Rebelo, J.M.M. 2015. Ants (Hymenoptera: Formicidae) associated with pig carcasses in an urban area. Sociobiology, 62(4): 527-532.

AntWeb. 2019. AntWeb. Available at: http://www.antweb.org. Access in: 03/01/2019.

AntWiki. 2019. AntiWiki. Available at: http://www.antwiki.org. Access in: 19/01/2019.

Bolton, B. 2019. An online catalog of the ants of the world. Available at: http:// www.antcat.org. Access in: 10/02/2019.

Borgmeier, T. 1955. Die Wanderameisen der neotropischen Region. Studia Entomologica, 3: 1-720.

Brandão, C.R.F. 1991. Adendos ao catálogo abreviado das formigas da região neotropical (Hymenoptera: Formicidae). Revista Brasileira de Entomologia, 35: 319-412.

Brandão, C.R.F.; Feitosa, R.M. \& Diniz, J.L.M. 2015. Taxonomic revision of the Neotropical Myrmicinae ant genus Blepharidatta Wheeler. Zootaxa, 4012(1): 33-56.

Brandão, C.R.F.; Silva, R.R. \& Feitosa, R.M. 2011. Cerrado ground-dwelling ants (Hymenoptera: Formicidae) as indicators of edge effects. Zoologia, 28(3): 379-387.

Brasil. 2009. Ministério do Meio Ambiente. Relatório técnico de monitoramento do desmatamento no bioma Cerrado, 2002 a 2008: dados revisados. Brasilia: MMA, 67p. Available at: http://www.mma.gov.br/estruturas/ sbf chm rbbio/ arquivos/relatorio tecnico monitoramento desmate bioma cerrado csr ibama 20022008 rev 72.pdf. Access in: 08/12/2018.

Carvalho, A.P.R.; Silva, C.G. \& Fonseca, A.R. 2011. Diversidade de formigas em um hospital público no município de Chapadinha, Maranhão, Brasil. Revista de Biologia e Ciências da Terra, 11: 67-73.

Celentano, D.; Rousseau, G.X.; Engel, V.L.; Zelarayán, M.; Oliveira, E.C.; Araújo, A.C.M. \& De Moura, E.G. 2017. Degradation of riparian forest affects soil properties and ecosystem services provision in eastern amazon of Brazil. Land Degradation \& Development, 28: 482-493.

Chaves, L.P.F.A.; Silva, R.A.; Amaral, Y.T.; Costa, M.K.L. \& Siqueira, G.M. 2016. Biogeographical diversity of north mesoregion of the Maranhão state (Brazil). Journal of Geospatial Modelling, 1: 19.

Cuezzo, F. 2000. Revisión del género Forelius (Hymenoptera: Formicidae: Dolichoderinae). Sociobiology, 35: 197-275.

Dalzochio, M.S.; Renner, S.; Sganzerla, C.; Prass, G.; Ely, G.J.; Salvi, L.C.; Dametto, N. \& Périco, E. 2018. Checklist of Odonata (Insecta) in the state of Rio Grande do Sul, Brazil with seven new records. Biota Neotropica, 18: 1-14. 
Dáttilo, W.; Vicente, R.E.; Nunes, R.V. \& Carvalho, M.S.G. 2010. First Record of the Quenquém cisco-da-Amazônia Acromyrmex hystrix (Latreille) (Formicidae: Myrmicinae) for Maranhão State, Brazil. EntomoBrasilis, 3(3): 92-93.

Dáttilo, W.; Vicente, R.E.; Nunes, R.V. \& Feitosa, R.M. 2012. Influence of cave size and presence of bat guano on ant visitation. Sociobiology, 59(2): 549-559.

De Andrade, M.L. \& Baroni Urbani, C. 1999. Diversity and Adaptation in the ant genus Cephalotes, past and present. Stuttgarter Beitrage zur Naturkunde Serie B, 271: 893.

Desidério, G.R.; Barcelos-Silva, P.; De Souza, W.R.M.; Pes, A.M. \& Azevêdo, C.A.S. 2017. Caddisflies (Insecta: Trichoptera) from Maranhão State, Northeast Region, Brazil: A new species, checklist, and new geographical records. Zootaxa, 4221: 151-171.

Dias, A.M. \& Lattke, J.E. 2019. A new species and new records of minutagroup Gnamptogenys from Brazil (Hymenoptera: Formicidae). Revista Brasileira de Entomologia, 63(1): 30-34.

Feitosa, R.M.; Brandão, C.R.F. \& Dietz, B.H. 2007. Basiceros scambognathus (Brown, 1949) n. comb., with the first worker and male descriptions, and a revised generic diagnosis (Hymenoptera: Formicidae: Myrmicinae). Papéis Avulsos de Zoologia, 47(2): 31-42.

Feitosa, R.M.; Brandão, C.R.F. \& Diniz, J.L.M. 2008. Revisionary studies on the enigmatic Neotropical ant genus Stegomyrmex Emery, 1912 (Hymenoptera: Formicidae: Myrmicinae), with the description of two new species. Journal of Hymenoptera Research, 17: 64-82.

Fernandes, I.0.; Oliveira, M.L. \& Delabie, J.H.C. 2014. Description of two new species in the Neotropical Pachycondyla foetida complex (Hymenoptera: Formicidae: Ponerinae) and taxonomic notes on the genus. Myrmecological News, 19: 133-163.

Figueiredo, C.J.; Silva, R.R.; Munhae, C.B. \& Morini, M.S.C. 2013. Fauna de formigas (Hymenoptera: Formicidae) atraídas a armadilhas subterrâneas em áreas de Mata Atlântica. Biota Neotropica, 13: 1-7.

Forel, A. 1904. Miscellanea myrmécologiques. Revue Suisse de Zoologie, 12: $1-52$.

Freitas, M.A.; Vieira, R.S.; Entiauspe-Neto, 0.M.; Sousa, S.0.; Farias, T.; Sousa, A.G. \& Moura, G.J.B. 2017. Herpetofauna of the Northwest Amazon forest in the state of Maranhão, Brazil, with remarks on the Gurupi Biological Reserve. ZooKeys, 643: 141-155.

Gasper, A.L.; Eisenlohr, P.V. \& Salino, A. 2016. Improving collection efforts to avoid loss of biodiversity: lessons from comprehensive sampling of lycophytes and ferns in the subtropical Atlantic Forest. Acta Botanica Brasilica, 30: 166-175.

Gonçalves, C.R. 1942. Contribuição para o conhecimento do gênero Atta Fabr., das formigas saúvas. Boletim da Sociedade Brasileira de Agronomia, 5: 333-358.

Gonçalves, C.R. 1947. Saúvas do sul e centro do Brasil. Boletim Fitossanitário, 2: 183-218.

Gonçalves, C.R. 1961. 0 gênero Acromyrmex no Brasil (Hym. Formicidae). Studia Entomologica, 4: 113-180.

Graham, C.H.; Elith, J.; Hijmans, R.J.; Guisan, A.; Townsend Peterson, A. \& Loiselle, B.A. 2008. The influence of spatial errors in species occurrence data used in distribution models. Journal of Applied Ecology, 45: 239-247.

Gutiérrez, J.A.M.; Rousseau, G.X.; Andrade-Silva, J. \& Delabie, J.H.C. 2017. Taxones superiores de hormigas como sustitutos de la riqueza de especies, en una cronosecuencia de bosques secundarios, bosque primario y sistemas agroforestales en la Amazonía Oriental, Brasil. Revista de Biología Tropical, 65(1): 279-291.

Hallmann, C.A.; Sorg, M.; Jongejans, E.; Siepel, H.; Hofland, N.; Schwan, H.; Stenmans, W.; Müller, A.; Sumser, H.; Hörren, T.; Goulson, D. \& de Kroon,
H. 2017. More than 75 percent decline over 27 years in total flying insect biomass in protected areas. Plos One, 12(10): e0185809.

Hijmans, R.J.; Garrett, K.A.; Huama'n, Z.; Zhang, D.P.; Schreuder, M. \& Bonierbale, M. 2000. Assessing the geographic representativeness of genebank collections: the case of Bolivian wild potatoes. Conservation Biology, 14: 1755-65.

Instituto Brasileiro de Geografia e Estatística (IBGE). 2011. Indice de Nomes Geográficos, Escala 1:1.000.000. Base Cartográfica Contínua do Brasil ao Milionésimo. Disponível em: http://www.ibge.gov.br/geociencias/ cartas-e-mapas/bases-cartograficas-continuas/15759-brasil. html?=\&t=sobre. Acesso em: 03/12/2018.

Instituto Brasileiro de Geografia e Estatística (IBGE). 2018. Maranhão. Available at: http://cidades.ibge.gov.br/brasil/ma/panorama. Access in: 02/02/2019.

Janicki, J.; Narula, N.; Ziegler, M.; Guénard, B. \& Economo, E.P. 2016. Visualizing and interacting with large-volume biodiversity data using client-server web-mapping applications: The design and implementation of antmaps. org. Ecological Informatics, 32: 185-193.

Jesovnik, A. \& Schultz, T.R. 2017. Revision of the fungus-farming ant genus Sericomyrmex Mayr (Hymenoptera, Formicidae, Myrmicinae). ZooKeys, 670: 1-109.

Johnson, R.A. 2015. A taxonomic revision of South American species of the seed-harvester ant genus Pogonomyrmex (Hymenoptera: Formicidae). Part I. Zootaxa, 4029(1): 1-142.

Kadmon, R.; Farber, 0. \& Danin, A. 2003. A systematic analysis of factors affecting the performance of climatic envelope models. Ecological Applications, 13: 853-67.

Kempf, W.W. 1959. Insecta Amapaensia. Hymenoptera: Formicidae. Studia Entomologica, 2: 209-218.

Kempf,W.W. 1960a. Insecta Amapaensia. Hymenoptera: Formicidae (segunda contribuição). Studia Entomologica, 3: 385-400.

Kempf, W.W. 1960b. Estudo sôbre Pseudomyrmex I. (Hymenoptera: Formicidae). Revista Brasileira de Entomologia, 9: 5-32.

Kempf, W.W. 1964. Uma nova Platythyrea do Brasil (Hym., Formicidae). Revista Brasileira de Entomologia, 11: 141-144.

Kempf, W.W. 1968. Miscellaneous studies on Neotropical ants. IV. (Hymenoptera, Formicidae). Studia Entomologica, 11: 369-415.

Kempf,W.W. 1971. A preliminary review of the ponerine ant genus Dinoponera Roger (Hymenoptera: Formicidae). Studia Entomologica, 14: 369-394.

Kempf, W.W. 1972a. Catálogo abreviado das formigas da região Neotropical (Hym. Formicidae). Studia Entomologica, 15: 1-4.

Kempf, W.W. 1972b. A new species of the Dolichoderine ant genus Monacis Roger, from the Amazon, with further remarks on the genus (Hymenoptera, Formicidae). Revista Brasileira de Biologia, 32: 251-254.

Kempf, W.W. 1975. Miscellaneous studies on neotropical ants. VI. (Hymenoptera, Formicidae). Studia Entomologica, 18: 341-380.

Lattke, J.E. 2011. Revision of the New World species of the genus Leptogenys Roger (Insecta: Hymenoptera: Formicidae: Ponerinae). Arthropod Systematics \& Phylogeny, 69: 127-264.

Leal, I.R.; Leal, L.; Oliveira, F.P.; Arcoverde, G.B. \& Andersen, A.N. 2017. Effects of human disturbance and climate change on myrmecochory in Brazilian Caatinga. In: Oliveira, P.S. \& Koptur, S. (Eds.). Ant-plant interactions. Impacts of human on terrestrial ecosystems. Cambridge, UK, Cambridge University Press. p. 112-132.

Lima, W.R.S.; Marques, S.G.; Rodrigues, F.S. \& Rebelo, J.M.M. 2013. Ants in a hospital environment and their potential as mechanical bacterial vectors. Revista da Sociedade Brasileira de Medicina Tropical, 46(5): 637-640.

Longino, J.T. \& Snelling, R.R. 2002. A taxonomic revision of the Procryptocerus (Hymenoptera: Formicidae) of Central America. Contributions in Science, 495: 1-30. 
Mann, W.M. 1916. The Stanford Expedition to Brazil, 1911, John C. Branner, director. The ants of Brazil. Bulletin of the Museum of Comparative Zoology, 60: 399-490.

Maracahipes-Santos, L.; Santos, J.0.; Reis, S.M. \& Lenza, E. 2018. Temporal changes in species composition, diversity, and woody vegetation structure of savannas in the Cerrado-Amazon transition zone. Acta Botanica Brasilica, 32(2): 254-263.

Ministério do Meio Ambiente (MMA). 2011. Plano de Ação para prevenção e controle do desmatamento e das queimadas: Cerrado. Brasília, MMA. 200p.

Monnin, R.; Ratnieks, F.L.W. \& Brandão, C.R.F. 2003. Reproductive Conflict in Animal Societies: Hierarchy Length Increases with Colony Size in Queenless Ponerine Ants. Behavioral Ecology and Sociobiology, 54(1): 71-79.

Moura, C.C.M.; Moura, G.J.B.; Lisboa, E.B.F. \& Luz, V.L.F. 2014. Distribuição geográfica e considerações ecológicas sobre a fauna de Testudines da Região Nordeste do Brasil. Sitientibus serie Ciencias Biologicas, 14: 1-20.

Newbold, T. 2010. Applications and limitations of museum data for conservation and ecology, with particular attention to species distribution models. Progress in Physical Geography, 34: 3-22.

Pereira, E.K.C.; Andrade-Silva, J.; Silva, 0.; Santos, C.L.C.; Moraes, L.S.; Bandeira, M.C.A.; Silva, C.R.R. \& Rebêlo, J.M.M. 2017. Solenopsis saevissima (Smith) (Hymenoptera: Formicidae) activity delays vertebrate carcass decomposition. Sociobiology, 64: 369.

Pereira, J.C.; Delabie, J.H.C.; Zanette, L.R.S. \& Quinet, Y. 2014. Studies on an Enigmatic Blepharidatta Wheeler Population (Hymenoptera: Formicidae) from the Brazilian Caatinga. Sociobiology, 61(1): 52-59.

Peres, C.A. 2005. Why we need mega-reserves in Amazonian forests. Conservation Biology, 19: 728-733.

QGIS Development Team. 2019. QGIS geographic information system. Open source geospatial foundation project. Disponível em: http://qgis.org/en/ site/forusers/download.html.

Ramos, A.S.J.C.; Lemos, R.N.S.; Vale, A.M.S; Batista, M.C.; Moreira, A.A.; Harada, H.Y. \& Mesquita, M.L.R. 2015. Ant diversity in agro ecosystems and secondary forest. African Journal of Agricultural Research, 10: 4449-4454.

Reddy, S. \& Dávalos, L.M. 2003: Geographical sampling bias and its implications for conservation priorities in Africa. Journal of Biogeography, 30: 1719-27.

Santos, B.F. \& Hoppe, J.P.M. 2018. Filling gaps in species distributions through the study of biological collections: 415 new distribution records for Neotropical Cryptinae (Hymenoptera, Ichneumonidae). Revista Brasileira de Entomologia, 62(4): 288-291.

Santos, G.M.M.; Delabie, J.H.C. \& Resende, J.J. 1999. Caracterização da Mirmecofauna (Hymenoptera: Formicidae) associada à vegetação periférica de inselbergs (Caatinga-arbórea-estacional-semi-descídua) em Itatim-Bahia-Brasil. Sitientibus. Revista da Universidade Estadual de Feira de Santana, 20: 33-43.

Santos, M.P.D.; Cerqueira, P.V. \& Soares, L.M.S. 2010. Avifauna em seis localidades no centro-sul do Estado do Maranhão, Brasil. Ornithologia, 4(1): 49-65.

Shoemaker, D.D.; Ahrens, M.E. \& Ross, K.G. 2006. Molecular phylogeny of fire ants of the Solenopsis saevissima species-group based on mtDNA sequences. Molecular Phylogenetics and Evolution, 38: 200-215.

Silva, E.F.; Corá, J.E.; Harada, A.Y. \& Sampaio, I.B.M. 2017. Association of the Occurrence of Ant Species (Hymenoptera: Formicidae) with Soil Attributes, Vegetation, and Climate in the Brazilian Cerrado Northeastern Region. Sociobiology, 64(4): 442-450.

Silva, G.M.; Carmo, M.S.; Moraes, L.S.; Moraes, F.C.; Barnabé, A.S. \& Figueiredo, P.M.S. 2012. Formigas (Hymenoptera: Formicidae) como Vetores de
Bactéria em Ambiente Hospitalar na Cidade de São Luis, Maranhão. Revista de Patologia Tropical, 41(3): 348-355.

Silva, P.R. 2007. Biologia de algumas espécies de Blepharidatta. Biológico, 69(2): 161-164.

Spinelli-Araujo, L.; Bayma-Silva, G.; Torresan, F.E.; Victoria, D.; Vicente, L.E.; Bolfe, E.L. \& Manzatto, C. 2016. Conservação da biodiversidade do estado do Maranhão: cenário atual em dados geoespaciais. Jaguariúna, EMBRAPA Meio Ambiente. 28p.

Stella, A. 2011. Plano de prevenção e controle do desmatamento e queimadas do Maranhão. São Luís, SEMA. 120p.

Ulysséa, M.A. \& Brandão, C.R.F. 2013. Ant species (Hymenoptera, Formicidae) from the seasonally dry tropical forest of northeastern Brazil: a compilation from field surveys in Bahia and literature records. Revista Brasileira de Entomologia, 57: 217-224.

Ulysséa, M.A.; Prado, L.P. \& Brandão, C.R.F. 2015. Type specimens of the traditional Myrmicinae (Hymenoptera: Formicidae) ant tribes deposited in the Museu de Zoologia da Universidade de São Paulo, Brazil: Adelomyrmecini, Basicerotini, Blepharidattini, Crematogastrini, Formicoxenini, Lenomyrmecini, Myrmicini, Phalacromyrmecini, Pheidolini, Stegomyrmecini, Stenammini and Tetramoriini. Papéis Avulsos de Zoologia, 55: 175-204. Disponível em: http://www.revistas. usp.br/paz/article/view/106374. Acesso em 25.04.2019.

Ulysséa, M.A.; Prado, L.P. \& Brandão, C.R.F. 2017. Catalogue of the Dolichoderinae, Formicinae and Martialinae (Hymenoptera: Formicidae) types deposited at the Museu de Zoologia da Universidade de São Paulo, Brazil. Papéis Avulsos de Zoologia, 57(23):295-311. Disponível em: http:// www.revistas.usp.br/paz/article/view/125647. Acesso em: 25.04 .2019

Vanzolini, P.E. 1992. A Supplement to the Ornithological Gazetteer of Brazil. São Paulo, Museu de Zoologia da Universidade de São Paulo. 252p.

Vanzolini, P.E. 2004. Episódios da Zoologia Brasílica. São Paulo, Hucitec. 212p.

Vanzolini, P.E. \& Papavero, N. 1968. Índice dos Topônimos Contidos na Carta do Brasil 1:1.000.000 do IBGE. São Paulo, Fundação de Amparo à Pesquisa do Estado de São Paulo. 201p.

Ward, P.S. 1989. Systematic Studies on Pseudomyrmecine Ants: Revision of the Pseudomyrmex oculatus and P. subtilissimus species groups with taxonomic comments on other species. Questiones Entomologicae, 25: 393-468.

Ward P.S. 1999. Systematics, biogeography and host plant associations of the Pseudomyrmex viduus group (Hymenoptera: Formicidae), Triplaris- and Tachigali-inhabiting ants. Zoological Journal of the Linnean Society, 126: 451-540.

Ward, P.S. 2007. The ant genus Leptanilloides: discovery of the male and evaluation of phylogenetic relationships based on DNA sequence data. Memoirs of the American Entomological Institute, 80: 637-649.

Ward, P.S. \& Downie, D.A. 2005. The ant subfamily Pseudomyrmecinae: phylogeny and evolution of big-eyed arboreal ants. Systematic Entomology, 30: 310-335.

Watkins, J.F. 1976. The identification and distribution of New World army ants (Dorylinae: Formicidae). Waco, Texas, Baylor University Press. 102p.

Wauters, N.; Dekoninck, W. \& Fournier, D. 2018. Introduction history and genetic diversity of the invasive ant Solenopsis geminata in the Galapagos Islands. Biological Invasions, 20(11): 3207-3226.

Wheeler, W.M. 1922. Observations on Gigantiops destructor Fabricius and other leaping ants. Biological Bulletin (Woods Hole), 42: 185-201.

Wild, A.L. 2007. Taxonomic revision of the ant genus Linepithema (Hymenoptera: Formicidae). University of California Publications in Entomology, 126: 1-151.

Wild, A.L. \& Cuezzo, F. 2006. Rediscovery of a fossil dolichoderine ant lineage (Hymenoptera: Formicidae: Dolichoderinae) and a description of a new genus from South America. Zootaxa, 1142: 57-68. 